\title{
Composición y diversidad de las comunidades de aves, moluscos y plantas asociadas con sistemas silvopastoriles de Matiguás y Río Blanco, Dpto. de Matagalpa, Nicaragua
}

A. Mijail Pérez ${ }^{1}$, Marlon Sotelo ${ }^{1,2}$, Freddy Ramírez ${ }^{1,2,}$ Iván Ramírez $^{1,2}$ Adolfo López ${ }^{1}$ e Isabel Siria ${ }^{2,1}$

\author{
1. Centro de Malacología y Diversidad Animal, UCA. Apto. 69, Managua, Nicaragua. \\ 2. Asociación Gaia \\ e-mail: ampp@ns.uca.edu.ni, mijail@ibw.com.ni
}

Recibido: octubre 2004/ Aceptado: noviembre 2004

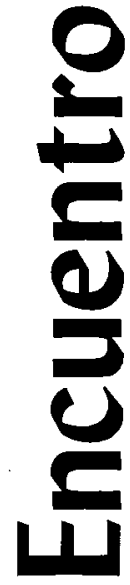

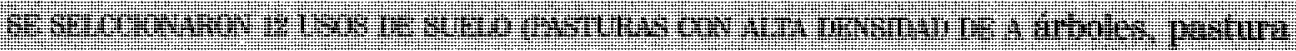

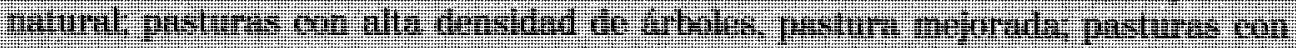

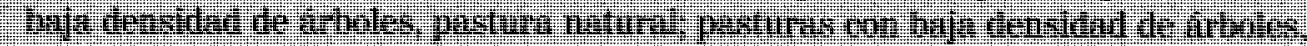

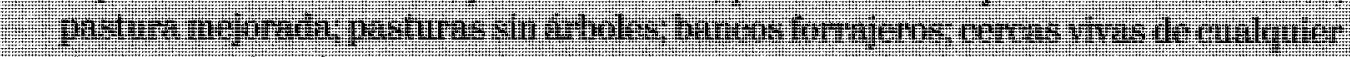

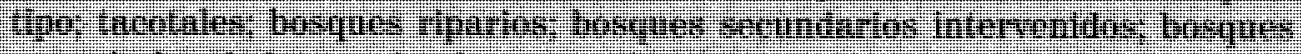

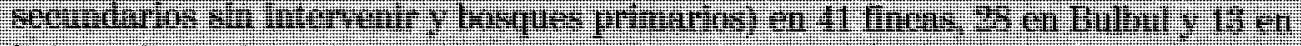

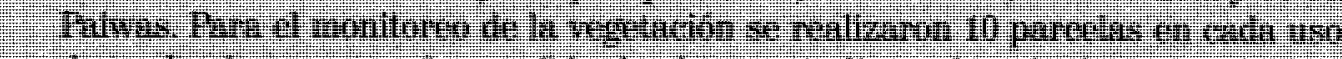

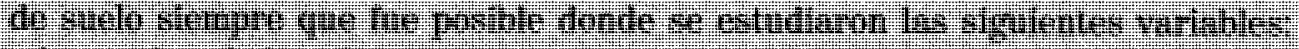

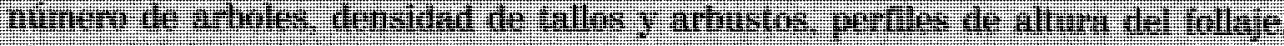

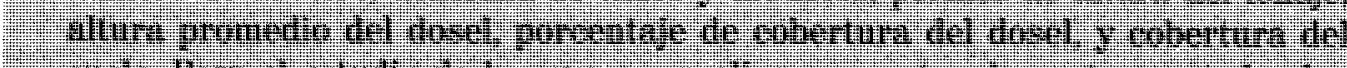

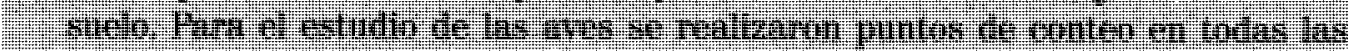

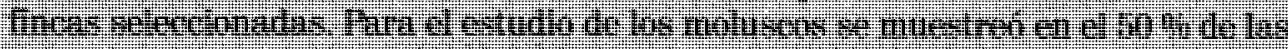

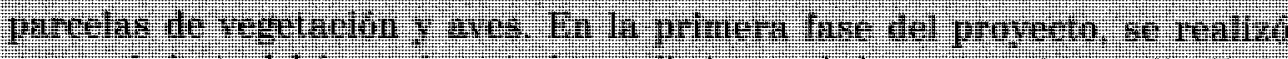

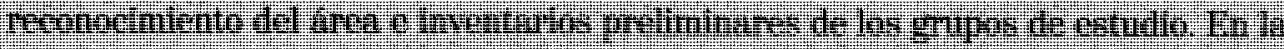

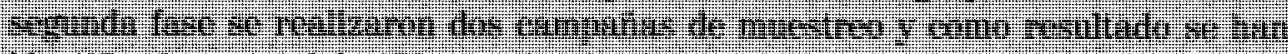

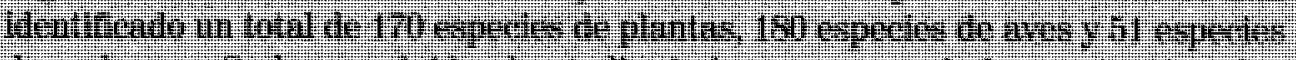

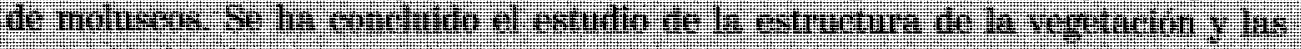

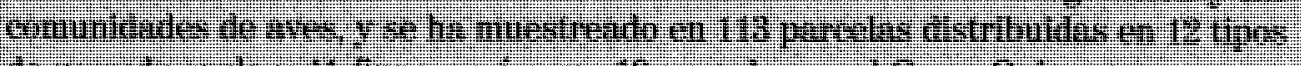

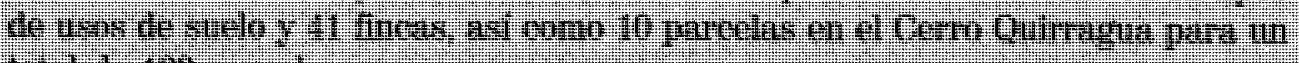

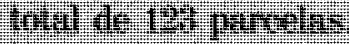




\section{Introducción}

Según Saunders et al., (1991) la fragmentación se puede definir como la transformación de un bosque continuo en muchas unidades más pequeñas y aisladas entre sí, cuya extensión agregada de superficie resulta ser mucho menor que la del bosque original. En este sentido, Marull y Marsallach (2002) plantearon que como respuesta a los fenómenos de fragmentación de los ecosistemas, surge la preocupación de mantener la conectividad ecológica entre los sistemas naturales. Por esta razón, numerosas estrategias y directivas nacionales e internacionales insisten en la necesidad de considerar los aspectos funcionales de los ecosistemas, especialmente la conectividad ecológica (Estrategia Global de Biodiversidad de 1992, Estrategia Paneuropea de diversidad biológica y paisajística de 1995, Estrategia de biodiversidad de la Comunidad Europea de 1998, etc.).

Los sistemas silvopastoriles son una modalidad de los sistemas agroforestales, donde se desarrollan árboles y pasturas manejados en forma conjunta, cuyo objetivo es incrementar la productividad en forma sostenible, supliendo además otros beneficios (Radulovich, 1994). Estudiar los componentes de cada sistema particular permitirá acercarse a la forma óptima de manejarlos. Afortunadamente cada vez existen más datos sobre diversidad de diferentes grupos biológicos en ecosistemas fragmentados y sistemas silvopastoriles (Estrada et al., 1997, Petit y Usher, 1998, Bergin et al., 2000, Fournier y Loreau, 2001, Ricketts et al., 2001, Estrada y Coates-Estrada, 2002, Jeanneret $e t$ al., 2003, Pérez, 2002, Weibull $e t a l ., 2003$, Naranjo, en línea),

Es interesante destacar que el área de estudio, los municipios de Matiguás y Paiwas, con una vocación eminentemente ganadera, constituye un escenario ideal para este tipo de proyectos. En el presente trabajo se presentan los resultados de un año de monitoreo sobre la composición y diversidad de aves, moluscos y vegetación en el área de estudio.

\section{Material y métodos}

Área de estudio: Se encuentra ubicada dentro del triángulo que conforman las reservas naturales, Sierra Quirragua, Cerro Musún y Fila Masigüe al sur (ver ilustración 1). Está compuesta por la comarca de Bulbul, perteneciente al Municipio de Matiguás, que se encuentra en las coordenadas UTM $670165 \mathrm{E}, 1417108 \mathrm{~N}$ y tiene una extensión de 1,335 km2 y una población de 38,584 habitantes, de los cuales el $81 \%$ vive en áreas rurales (INEC, 1995; Levard et al., 2001), así como por la comarca de Paiwas, perteneciente al Municipio de Río Blanco, que se encuentra en las coordenadas UTM $686152 \mathrm{E}, 1424706 \mathrm{~N}$ y tiene una extensión de $700 \mathrm{~km} 2$ y una población de 33,195 habitantes, de los cuales 23,950 (72.15\%) vive en áreas rurales (INIFOM, en línea); ambos pertenecientes al departamento de Matagalpa. 


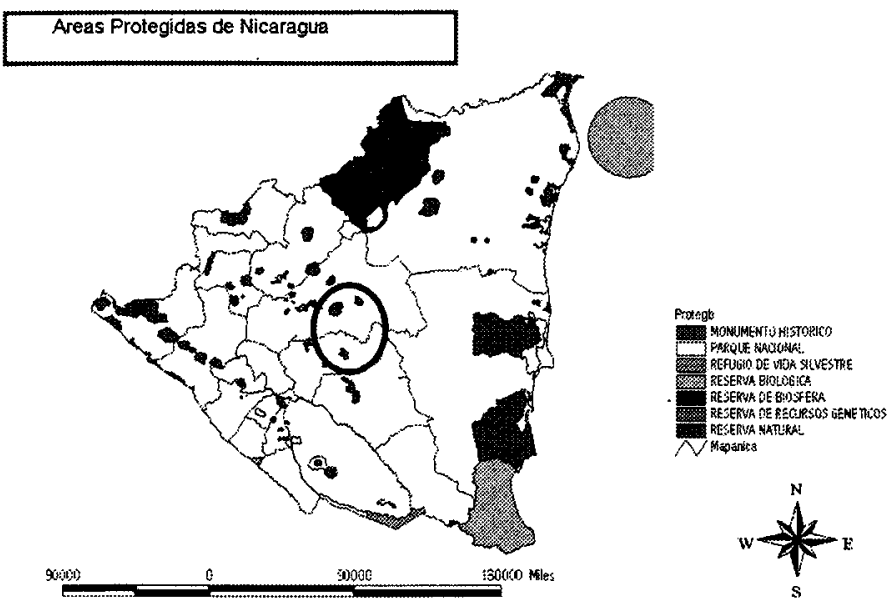

Ilustración 1. El área de estudio comprendida dentro del triángulo compuesto por las reservas naturales, Sierra Quirragua, Cerro Musún y Fila Masigüe al sur. Mapa tomado de CBM-MARENA (2001).

Selección de las fincas y los usos: Se seleccionaron fincas con la mayor cantidad de usos de suelo posible y de ese primer grupo se realizó una segunda selección de aquellas fincas en las que los usos del suelo existentes tuviesen un área mayor que una manzana.

Se trabajó en 12 usos del suelo: pasturas con alta densidad de árboles, pasturas naturales; pasturas con alta densidad de árboles, pasturas mejoradas; pasturas con baja densidad de árboles, pasturas naturales; pasturas con baja densidad de árboles, pasturas mejoradas; pasturas sin árboles; banco forrajero de leñosas; cercas vivas; tacotales; bosques riparios; bosques secundarios intervenidos; bosques secundarios sin intervenir; bosques primarios. En cada uso del suelo se hicieron 10 réplicas siempre que fue posible. Se evitó hacer repeticiones de un mismo tipo de uso en una finca particular, por lo que, si existió la posibilidad de tener un mismo tipo de hábitat en la finca, la parcela a evaluar se escogió al azar.

El centro de las parcelas de muestreo se ubicó aproximadamente en el centro del uso del suelo con lo que se espera haber minimizado el efecto de borde en la misma. El centro de la parcela de observación de aves coincide con el centro de la parcela de evaluación de vegetación.

Vegetación: Como fase preliminar del estudio se realizó un inventario de las especies arbóreas más abundantes en la zona de estudio. Para la identificación se utilizaron las obras de Salas (1993) y de Poveda y Sánchez-Vindas (1999). Para estudiar la diversidad estructural de la vegetación se utilizó un método compuesto por aportes de James y Shugart (1970), Noon (1981), Schemske y Brokaw (1981), y Wunderle y Waide (1993), según Chipley et al., (2003).

En cada parcela estudiada se tomaron datos de una serie de parámetros como el Dosel, la Cobertura Basal, el diámetro DAP (por encima de $1.3 \mathrm{~m}$ ) según una escala de intervalos, y la altura de la vegetación, todo ello haciendo un recorrido a lo largo de una cuerda en forma de cruz, dividida en 4 brazos y con 5 puntos en cada brazo. Un árbol fue considerado cuando tuvo más de $3 \mathrm{~cm}$ de DAP (ver ilustración 2) 


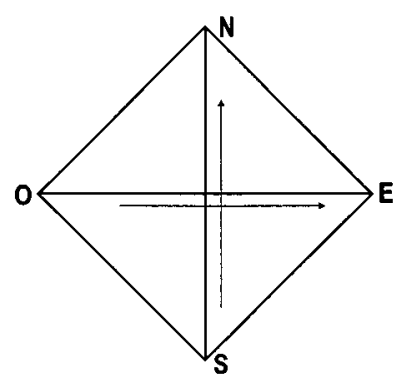

llustración 2. Parcela de monitoreo de la estructura de la vegetación.

Al final de las mediciones se tomaron fotos de cada uno de los puntos cardinales extremos de la cuerda, de cara a documentar los posibles cambios que tendrán lugar en el año 3 y en el 5 .

Cercas vivas: Se realizó una modificación en la forma de las parcelas en la toma de datos de estructura de la vegetación para hacerla más acorde con la forma y estructura de la cerca viva. Para ello la muestra se tomó en una parcela de 200 metros de largo por 2 metros de ancho (0,04 ha o $400 \mathrm{~m} 2)$.

En cada parcela se contó el número de árboles presentes, a los cuales se les asignó una escala de siete categorías de diámetro a la altura del pecho (DAP, 1,3 m), la asignación de cada categoría se realizó por medio de una regla marcada así: $\mathrm{S}:>3-8 \mathrm{~cm}$., A: $>8-15 \mathrm{~cm}$., $\mathrm{B}$ : $>15-23 \mathrm{~cm} ., \mathrm{C}:>23-38 \mathrm{~cm} ., \mathrm{D}:>38-53 \mathrm{~cm} ., \mathrm{E}:>53-69 \mathrm{~cm} ., \mathrm{F}:>69-84 \mathrm{~cm}$.

La densidad del follaje se midió en 20 puntos a lo largo de la cerca viva, uno cada $10 \mathrm{~m}$. La medición de la densidad se evaluó en categorías de altura de $0.5 \mathrm{~m}$, cuantificando la presencia o ausencia de follaje de acuerdo con la cantidad de follaje vivo que tocó cada categoría mencionada en un tubo de $3.0 \mathrm{~m}$ colocado verticalmente en cada punto. Las categorías superiores a $3 \mathrm{~m}$ fueron estimadas a lo largo del tubo y la presencia o ausencia de follaje en cada intervalo usando un rangefinder óptico, por encima de $6 \mathrm{~m}$ los intervalos fueron: $3-4 \mathrm{~m}$, 4-6 m, 6-8 m, 10-12 m, 12-15 m, y 15-20 m.

La densidad de arbustos con DAP $3 \mathrm{~cm}$., fue estimada al contar el número de éstos que tocaron al observador a la altura del pecho, al caminar con sus brazos abiertos a lo largo de los $200 \mathrm{~m}$ de la cerca viva (desde que el observador se quede a un lado u otro de la cerca, será necesario extrapolar como si fuera caminando a lo largo de una línea dentro de la cerca).

Bosques riparios: Cuando los bosques riparios fueron estrechos se adoptó la metodología usada para cercas vivas.

Aves: Como fase preliminar del estudio se realizó un inventario de las especies de aves más abundantes en la zona de estudio. Para el estudio estructural de las aves se realizaron muestreos en las fincas seleccionadas. Los observadores realizaron los muestreos entre $6 \mathrm{y}$ 10 de la mañana. 
Las observaciones se realizaron en puntos de conteo de 25 metros de radio durante 10 minutos, haciendo la observación desde el centro de la parcela como sugiere Wunderle (1994) (ver ilustración 3). Cada vez que se llegó a un punto de conteo se dejó pasar 5 minutos con el fin de que las aves se acostumbrasen a nuestra presencia.

Para la identificación y los datos generales de las aves se utilizaron las guías de Howell y Webb (1995) y Stiles y Skutch (1998), así como la lista patrón de la AOU (1998), para la ubicación sistemática de las especies.

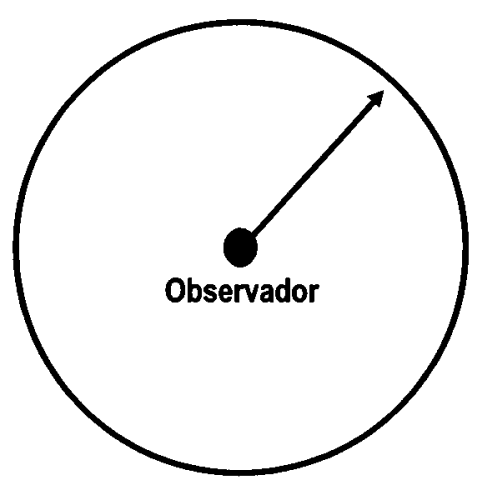

Ilustración 3. Punto de monitoreo de la estructura de las comunidades de aves.

Las campañas de muestreo fueron como sigue:

Campaña 1: diciembre 2003- febrero 2004

Campaña 2: abril- mayo 2004

Campaña 3: julio- agosto 2004

Moluscos: Para su estudio se utilizaron los mismos puntos que para la observación de aves. La recolecta se realizó como habitualmente para este grupo (Altonaga, 1988 y Puente, 1994). Se muestreó en el $50 \%$ de las parcelas definidas para el muestreo de la estructura de la vegetación y aves. El muestreo se efectuó mediante el levantamiento de piedras, revisión ocular de la hojarasca y/o el mantillo, cortezas y oquedades de árboles, troncos, etc. En cada parcela se recogió una muestra de mantillo de un área aproximada de $50 \times 50 \mathrm{~cm}$., con el objetivo de no pasar por alto los microgasterópodos; las muestras se introdujeron en bolsas de plástico etiquetadas que fueron llevadas al laboratorio, donde se separó e identificó el material.

Los muestreos fueron realizados por dos personas durante una hora para un total de 65 parcelas. Se realizaron recolectas en época seca y se realizarán en la época de lluvia. Toda la separación se realizó a mano bajo microscopios estereoscópicos Wild y/o Nikon. Los ejemplares se depositaron en cápsulas de plástico debidamente etiquetadas y luego dentro de tubos de cristal. Para la identificación del material se utilizó la obra de Pérez y López (2002), así como la clave electrónica de diagnóstico de Pérez y Torres (2002). Para complementar se revisaron las colecciones del Centro de Malacología/ Diversidad Animal de la Universidad Centroamericana. 
Análisis de los datos: Se brindan los datos de riqueza y abundancia para cada comunidad en cada uso de suelo. En este sentido:

S: Riqueza de especies. Es considerado el índice más sencillo de diversidad.

A: Abundancia. Cantidad de individuos de una especie determinada en una comunidad.

Partiendo de los datos de riqueza y abundancia, se confeccionaron los índices ecológicos H' diversidad de Shannon y Weaver (1949) y $\lambda$ de Simpson (1949), así como $E_{1}$ y $E_{5}$, Equitatividad de Pielou (1977) y Hill (1973) respectivamente.

Índice de Shannon y Weaver (1949):

$\mathrm{S}$

$$
H^{\prime}=-\sum_{i=1}\left(p_{i} \operatorname{Ln} p_{i}\right) \text { donde: }
$$

$\mathrm{p}_{\mathrm{i}}=\mathrm{es}$ la abundancia proporcional de la ith especie, la cual está dada por la fórmula:

$\mathrm{p}_{\mathrm{i}}=\mathrm{n}_{\mathrm{i}} \mathrm{N}, \quad \mathrm{i}=1,2,3, \ldots \mathrm{S}$. Donde:

$\mathrm{n}_{\mathrm{i}}=$ no. de individuos de la ith especie

$\mathrm{N}=$ no. total de individuos conocidos para todas las especies en la población.

Ïndice de Simpson (1949):

$$
\lambda=\sum_{i=1}^{S} p_{i}^{2}
$$

Donde los componentes significan lo mismo que en el índice de Shannon-Weaver.

Índice $E_{1}$ (Ludwig y Reynolds, 1988): $E_{1}=J^{\prime}=H^{\prime} / \ln (S)=\ln \left(\mathrm{N}_{1}\right) / \ln (\mathrm{NO})$

Este índice expresa $\mathrm{H}^{\prime}$ relacionada con el máximo valor que $\mathrm{H}^{\prime}$ puede obtener cuando todas las especies de la muestra son perfectamente equitativas con un individuo por especie.

Indice $\mathrm{E}_{5}$ (Ludwig y Reynolds, 1988): $\mathrm{E}_{5}=(1 / \lambda) 1 / \mathrm{e}^{\mathrm{H}^{\prime}} 1=\mathrm{N}_{2} 1 / \mathrm{N}_{1}-1$

E5 se aproxima a cero cuando una especie se vuelve más dominante en la comunidad, la cual es una propiedad muy deseada por un índice de equitatividad (Pérez, 2004).

Para realizar los análisis de clasificación se empleó el Coeficiente de Jaccard (1901) según una estrategia de ligamiento simple. La expresión del citado coeficiente es: $\mathrm{CJ}=\mathrm{c} / \mathrm{c}+\mathrm{a}+\mathrm{b}$ Donde:

$\mathrm{c}=$ número de especies comunes para las dos muestras

$\mathrm{a}=$ número de especies de la muestra $\mathrm{A}$

$\mathrm{b}=$ número de especies de la muestra $\mathrm{B}$

Para los tres grupos taxonómicos estudiados se calcularon las curvas de saturación de especies según Colwell y Coddington (1994). Los índices empleados fueron los siguientes:

Índice de Chao1: 
$S_{1}=S_{o b s}+\frac{F_{1}^{2}}{2\left(F_{2}+1\right)}-\frac{F_{1} F_{2}}{2\left(F_{2}+1\right)^{2}}$

Índice de $\mathrm{Chao}_{2}$ :

$\mathrm{S}_{2}=\mathrm{S}_{\mathrm{obs}}+\frac{\mathrm{Q}_{1}{ }^{2}}{2\left(\mathrm{Q}_{2}+1\right)}-\frac{\mathrm{Q}_{1} \mathrm{Q}_{2}}{2\left(\mathrm{Q}_{2}+1\right)^{2}}$

Donde:

$\mathrm{S}_{\mathrm{obs}}$ : Número total de especies observadas en todas las muestras reunidas.

Fi: Número de especies que tienen exactamente i individuos cuando todas las muestras están agrupadas $\left(\mathrm{F}_{1}\right.$ es el número de singletons o especies representadas por un individuo y $\mathrm{F}_{2}$ es el número de doubletons o especies representadas por dos individuos).

142 Qj: Número de especies que tienen exactamente $\mathrm{j}$ individuos cuando todas las muestras están agrupadas $\left(Q_{1}\right.$ es el número de únicos o especies representadas por un individuo y $_{2}$ es el número de duplicados o especies representadas por dos individuos).

En el caso de las aves, la información de cuáles especies se encontraban amenazadas, vulnerables o en peligro de extinción se obtuvo de CCAD (1999). Las categorías de dependencia del bosque fueron seguidas según Stiles y Skutch (1998).

Se realizaron varios análisis estadísticos según Sokal y Rohlf (1981) para probar relaciones entre las abudancias, riquezas de especies, relación entre la diversidad estructural del follaje y la diversidad de especies de la vegetación, entre otros. Los mismos se realizaron usando el programa SPSS.

Abreviaturas: Las abreviaturas significan lo siguiente:

- Pasturas con alta densidad de árboles, pastura natural (PNADA)

- Pasturas con alta densidad de árboles, pastura mejorada (PMADA)

- Pasturas con baja densidad de árboles, pastura natural (PNBDA)

- Pasturas con baja densidad de árboles, pastura mejorada (PMBDA)

- Pasturas sin árboles (PSA)

- Bancos forrajeros de leñosas (BFL)

- Cercas vivas (CV)

- Tacotales (TAC)

- Bosques riparios (BR)

- Bosques secundarios intervenidos (BSI)

- Bosques secundarios sin intervenir (BS)

- Bosques primarios (BP)

- Bosque primario Quirragua (BPQ=BPP) 


\section{Resultados}

\section{Fincas:}

Se muestreó en 123 parcelas, de las cuales 113 están distribuídas en 12 tipos de usos de suelo y 41 fincas, 28 en Bulbul y 13 en Paiwas. Las otras 10 parcelas corresponden a bosque primario y fueron realizadas en el Cerro Quirragua.

\section{Vegetación:}

Composición de especies: Se observó un total de 170 especies distribuidas en 57 familias. Como se puede observar en la ilustración 4, en el área de estudio predominan las familias Caesalpinaceae, Fabaceae y Mimosaceae. La familia Burseraceae está representada por una sola especie, Bursera simarouba, pero esta especie es la más abundante de todas las presentes en los sistemas, por lo que se incluye esta familia entre las más diversas de las encontradas en la zona.

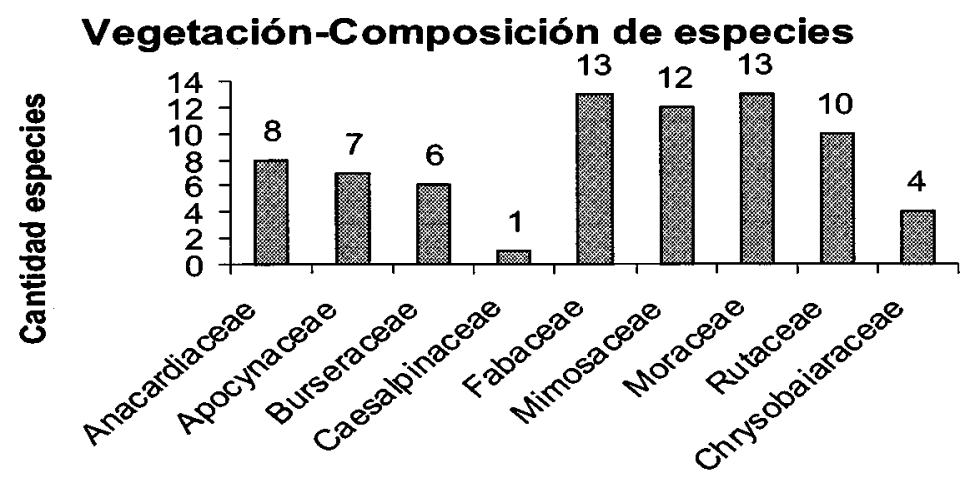

Ilustración 4. Cantidades de especies por familias

Estructura de las comunidades: En la ilustración 5, se muestran las curvas de saturación de especies según el índice de Chao 1. Como puede verse, el número de especies observadas todavía no forma una curva asintótica, lo que indica que aún hay especies por encontrar en la comunidad; para el índice de Chao 1 serían 173 especies y para el de Chao 2, 189 especies.

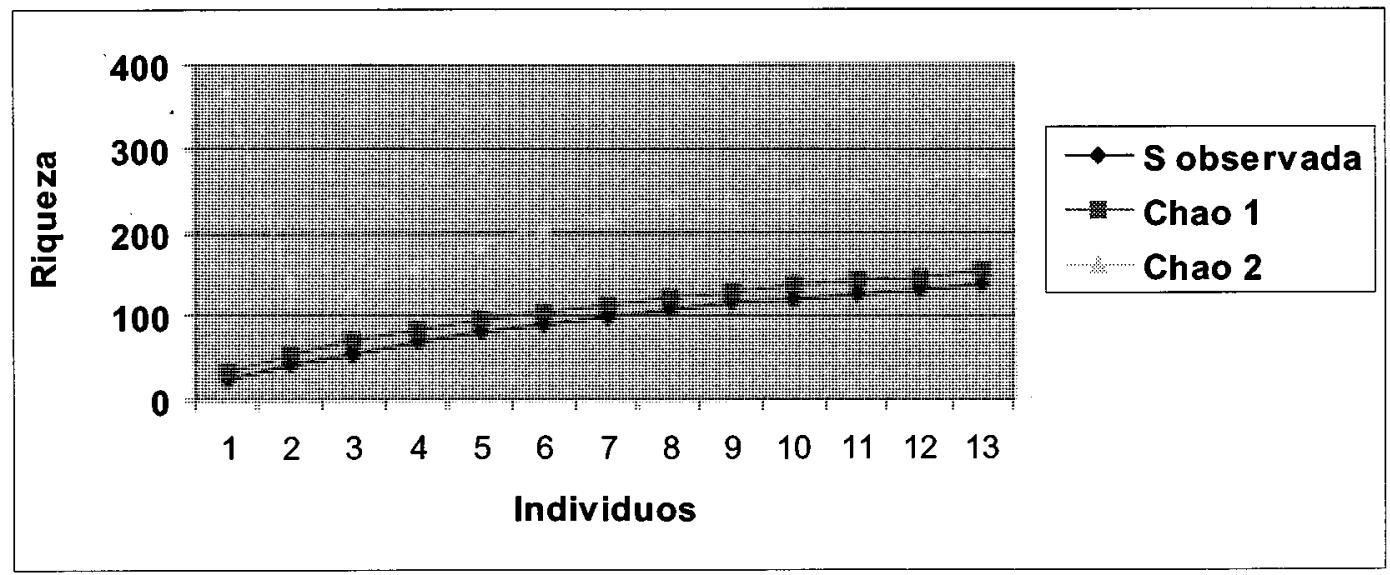

llustración 5. Curvas de saturación de especies de árboles 
Los índices ecológicos muestran los valores más altos de riqueza de especies en el bosque primario de Quirragua $(S=46)$ seguidos por los bosques riparios de los sistemas $(S=43)$ (ver cuadro 1).

Cuadro 1. Índices ecológicos estructurales calculados en la vegetación de los 12 usos de suelo en los sistemas silvopastoriles de los municipios de Matiguás y Río Blanco

\begin{tabular}{|l|c|c|c|c|c|c|}
\hline Usos & S & A & $\lambda$ & $H^{\prime}$ & E1 & E5 \\
\hline PNADA & 21 & 170 & 0.19 & 2.14 & 0.70 & 0.59 \\
\hline PMADA & 17 & 174 & 0.22 & 1.99 & 0.70 & 0.57 \\
\hline PNBDA & 19 & 100 & 0.16 & 2.15 & 0.73 & 0.70 \\
\hline PMBDA & 14 & 54 & 0.40 & 1.57 & 0.59 & 0.40 \\
\hline PNSA & 1 & 0 & 0 & 0 & 0 & 0 \\
\hline BFL & 21 & 210 & 0.15 & 2.26 & 0.74 & 0.64 \\
\hline CV & 34 & 825 & 0.27 & 2.09 & 0.59 & 0.39 \\
\hline TAC & 23 & 174 & 0.14 & 2.38 & 0.76 & 0.62 \\
\hline BR & 43 & 270 & 0.14 & 2.63 & 0.70 & 0.49 \\
\hline BSI & 38 & 247 & 0.11 & 2.74 & 0.75 & 0.56 \\
\hline BS & 32 & 153 & 0.12 & 2.72 & 0.78 & 0.53 \\
\hline BP & 30 & 77 & 0.04 & 3.12 & 0.92 & 0.98 \\
\hline BPQ & 46 & 294 & 0.04 & 3.34 & 0.87 & 0.71 \\
\hline
\end{tabular}

Análisis de agrupamiento: El análisis de agrupamiento (ver ilustración 6) en la vegetación arbórea muestra un comportamiento algo complejo, posiblemente debido al manejo que sufre este grupo por parte de los productores. Se presentan cuatro usos que no se agrupan con los otros, estos son las PNSA, BP, BSI y bosque primario de Quirragua. El resto de los usos conforma dos grupos, uno compuesto por BR, CV y BS y el otro por BFL, PMBDA, PMADA, PNADA, TAC y PNBDA.

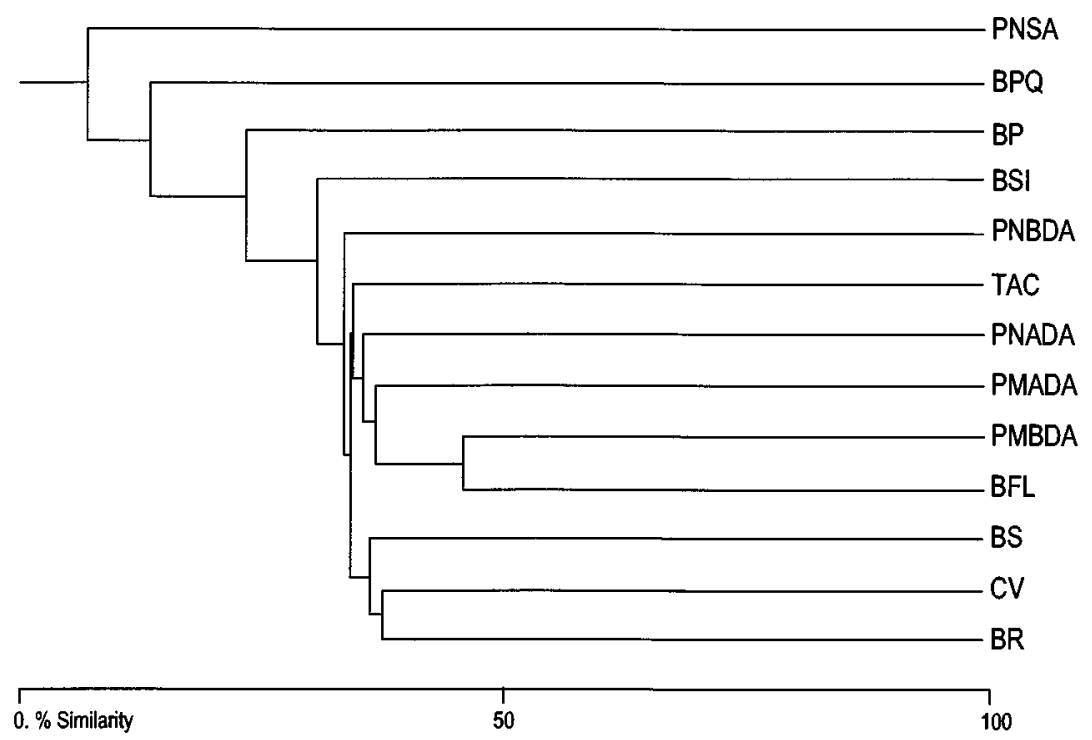

Ilustración 6. Dendrograma de similitud de los tipos de uso en relación con la presencia o ausencia de especies de árboles 


\section{Estructura de la vegetación:}

En el marco del presente artículo sólo se han analizado los perfiles de altura de follaje (ver ilustración 7), del total de variables de la estructura de la vegetación, debido a que posiblemente esta sea la variable más relacionada con la biodiversidad como proponen MacArthur y MacArthur (1961).

liustración 7. Perfil de altura de follaje

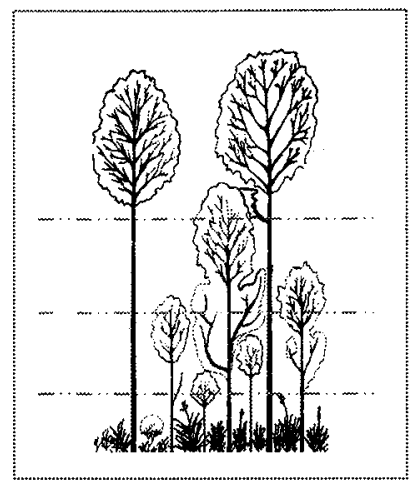

Perfil de altura de follaje: Como se aprecia en el cuadro 2, en las pasturas existe un mayor porcentaje de vegetación en los estratos comprendidos entre 0 y $1 \mathrm{~m}$ así como entre 3 y $10 \mathrm{~m}$. Por el contrario, en los usos relacionados a recursos forestales como BFL, TAC, BP, BS, BSI, BR y CV la vegetación presente entre los diferentes estratos es más homogénea.

Cuadro 2. Perfil de altura de follaje

\begin{tabular}{|c|c|c|c|c|c|c|c|c|c|c|c|c|c|c|c|}
\hline & \multicolumn{15}{|c|}{ Intervalos de altura del follaje } \\
\hline & $0-0.25$ & $0.25-0.5$ & $0.5-0.75$ & $0.75-1$ & $1-1.5$ & $1.5-2$ & $2-2.5$ & $2.5-3$ & $3-4$ & $4-6$ & $6-8$ & $8-10$ & $10-12$ & $12-15$ & $15-20$ \\
\hline PSA & 96.5 & 43.5 & 15.5 & 2 & 0.5 & 0.5 & 0 & 0 & 0 & 0 & 0 & 0 & 0 & 0 & 0 \\
\hline PNADA & 95.0 & 40.0 & 11.0 & 3.0 & 0.5 & 2.0 & 4.0 & 8.0 & 20.5 & 33.0 & 21.5 & 9.5 & 6.5 & 6.0 & 11.5 \\
\hline PNBDA & 98.5 & 48.5 & 18.5 & 6.5 & 3.0 & 0.0 & 2.0 & 7.5 & 10.5 & 7.0 & 7.5 & 9.5 & 7.0 & 7.5 & 8.0 \\
\hline PMBDA & 97.5 & 60.5 & 38.5 & 18.0 & 9.5 & 1.0 & 1.5 & 4.5 & 9.0 & 6.0 & 3.0 & 4.0 & 1.0 & 2.0 & 2.0 \\
\hline BFL & 84.0 & 40.5 & 16.5 & 8.0 & 6.5 & 2.0 & 4.5 & 7.5 & 22.0 & 26.0 & 30.0 & 15.0 & 5.5 & 1.5 & 3.5 \\
\hline PMADA & 93.5 & 64.0 & 35.0 & 14.0 & 5.5 & 0.5 & 1.5 & 3.0 & 10.0 & 16.0 & 14.0 & 13.0 & 9.5 & 6.0 & 3.5 \\
\hline BR & 57.0 & 32.0 & 15.0 & 5.5 & 6.0 & 8.0 & 14.0 & 15.5 & 37.5 & 29.0 & 34.5 & 30.0 & 23.0 & 21.0 & 23.0 \\
\hline BSI & 68.5 & 19.0 & 8.5 & 7.0 & 10.5 & 13.0 & 12.5 & 13.5 & 31.0 & 28.0 & 32.0 & 30.5 & 17.5 & 14.5 & 23.0 \\
\hline BP & 50.0 & 10.0 & 10.0 & 4.2 & 6.7 & 5.8 & 11.7 & 16.7 & 22.5 & 22.5 & 22.5 & 32.5 & 13.3 & 10.8 & 40.0 \\
\hline TAC & 64.5 & 32.0 & 20.5 & 16.5 & 19.0 & 17.5 & 21.0 & 14.5 & 22.0 & 21.0 & 10.5 & 6.5 & 2.0 & 2.0 & 0.0 \\
\hline BS & 42.9 & 12.1 & 9.3 & 4.3 & 9.3 & 10.7 & 12.1 & 12.9 & 23.6 & 26.4 & 27.9 & 22.1 & 12.1 & 12.1 & 40.0 \\
\hline$B P Q$ & 11.0 & 19.0 & 19.0 & 17.0 & 20.5 & 23.0 & 20.5 & 25.5 & 34.5 & 43.0 & 30.5 & 33.5 & 23.0 & 23.5 & 70.0 \\
\hline CV & 85.5 & 30.5 & 14.0 & 6.0 & 9.0 & 5.0 & 7.5 & 13.0 & 39.0 & 39.5 & 20.5 & 10.5 & 6.5 & 9.5 & 9.5 \\
\hline
\end{tabular}

\section{Aves:}

Composición de especies: Se observó un total de 180 especies distribuidas en 39 familias y 14 órdenes. De esta cantidad, en las parcelas de muestreo (plots) se registraron 151 especies, 33 familias y 13 órdenes. De las 151 especies contadas en los puntos de conteo, 98 especies fueron contadas en la primera campaña, 107 en la segunda y 94 en la tercera. Las familias con mayor número de especies en el área de estudio son Tyrannidae (25 especies), Parulidae (18 especies) y Thraupidae (14 especies) (ver ilustración 8). 


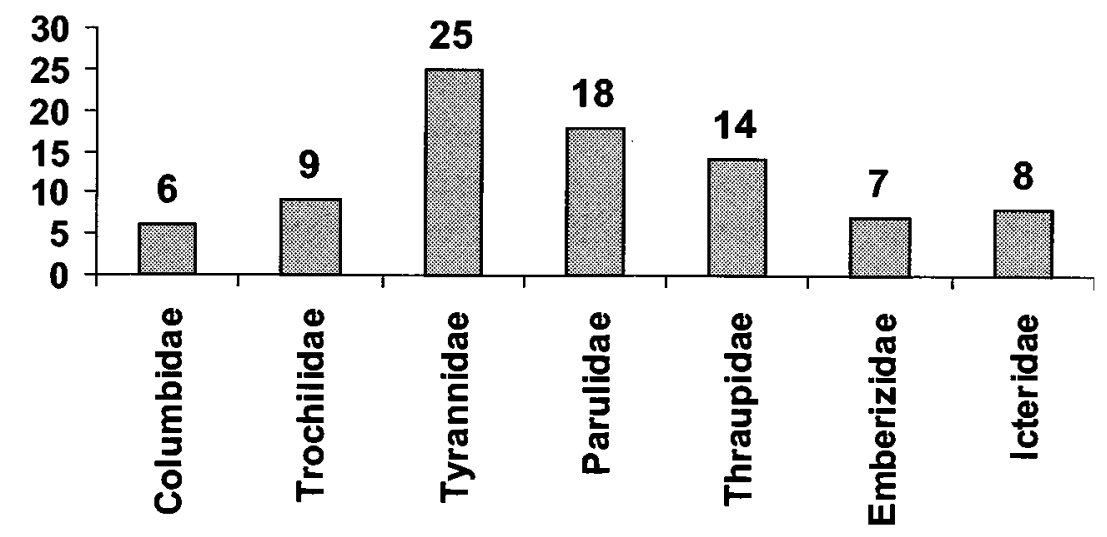

Ilustración 8. Cantidades de especies por familias

Especies amenazadas o vulnerables: Se encontraron 27 especies de aves en alguna de las categorías de amenaza de la CCAD (1999).

Cuadro 3. Especies que se encuentran en algún tipo de amenaza

\begin{tabular}{|l|l|l|l|l|l|l|}
\hline \multicolumn{1}{|c|}{ NOMBRE CIENTIFICO } & 1 & 2 & 3 & 4 & 5 & 6 \\
\hline Elanoides forficatus & & & & & & \\
\hline Asturina nitida & & & & & & \\
\hline Caracara plancus & & & & & & \\
\hline Herpetotheres cachinna & & & & & \\
\hline Ortalis cinereiceps & & & & & \\
\hline Aratinga nana & & & & & \\
\hline Brotogeris jugularis & & & & & \\
\hline Amazona albifrons & & & & & \\
\hline Amazona autumnalis & & & & & \\
\hline Tyto alba & & & & & \\
\hline Pseudoscops clamator & & & & & \\
\hline Otus cooperi & & & & & \\
\hline Amazilia rutila & & & & & \\
\hline Amazilia tzacatl & & & & & \\
\hline Amazilia cyanura & & & & & & \\
\hline Amazilia saucerrotei & & & & & & \\
\hline Archilocus colubris & & & & & & \\
\hline Eupherusa eximia & & & 3 & 4 & 9 & 0 \\
\hline Phaethornis longuemareus & & & & & & \\
\hline Phaethornis superciliosus & & & & & & \\
\hline Anthracothorax prevostii & & & & & & \\
\hline Pteroglossus torquatus & & & & & & \\
\hline Ramphastos sulfuratus & & & & & & \\
\hline Calocitta formosa & & & & & \\
\hline Turdus grayi & & & & & & \\
\hline Icterus galbula & & & & & & \\
\hline Psaracolius montezuma & & & & & & \\
\hline & & & & & & \\
\hline
\end{tabular}


Leyenda: 1- Especies que se encuentran en el Apéndice II de CITES; 2- Especies que se encuentren en el Apéndice III de CITES, 3- Lista de especies nicaragüenses de Aves silvestres con valor comercial no incluidas en Apéndices CITES y que requieren permiso de exportación, 4- Especies de aves silvestres protegidas de Nicaragua de 1998. Vedas indefinidas de aves silvestres, 5- Especies de aves silvestres protegidas de Nicaragua de 1998. Vedas parciales de aves silvestres, 6- Lista Roja Global para Nicaragua.

Estructura de las comunidades: En la ilustración se muestran las curvas de saturación de especies según los índices de Chao1 y Chao2. Los mismos muestran un comportamiento que todavía no es asintótico, lo que significa que existen nuevas especies a encontrar en la zona (ver ilustración 9), el índice de Chao1 predice una cantidad de 153 especies a esperar, y el índice de Chao2, 275 especies.

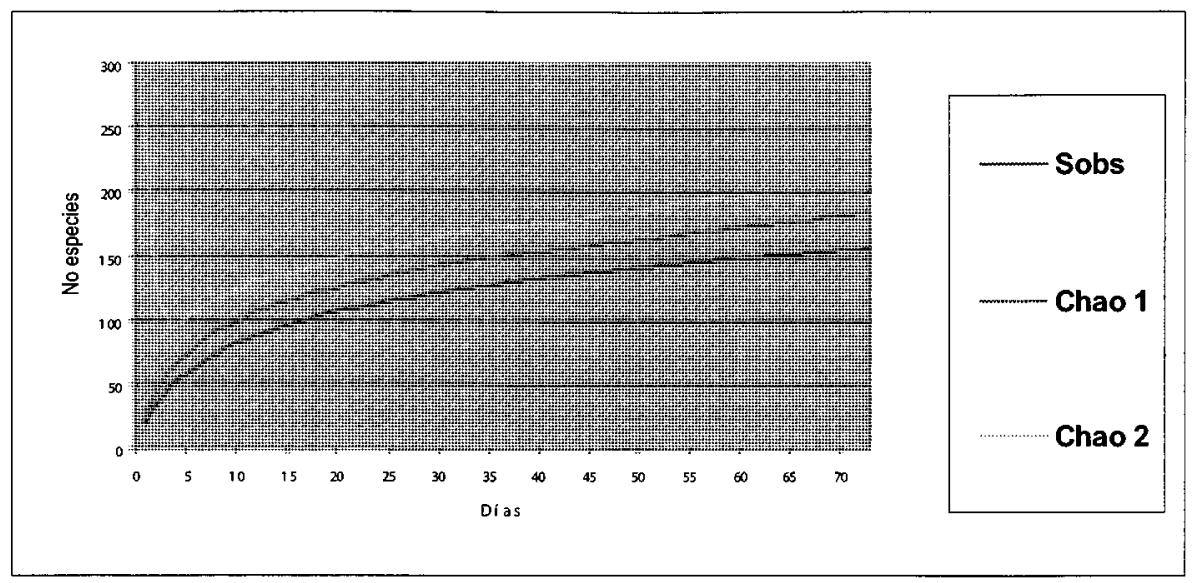

llustración 9. Curvas de saturación de especies de aves

Los valores de los índices estructurales indican que las comunidades más ricas se presentan en las cercas vivas seguidas por las pasturas mejoradas con alta densidad de árboles (ver cuadro 4), durante la primera campaña de muestreo, en cambio durante la segunda y la tercera campaña, los valores más altos se registraron en los bosques riparios (ver cuadros 5 у 6).

$\mathrm{Al}$ analizar los índices de diversidad (Shannon) entre los diferentes usos de suelos para cada una de las campañas, encontramos que en la primera campaña los dos valores más altos se presentaron en las pasturas naturales con baja densidad de árboles $\left(\mathrm{H}^{\prime}=3.35\right)$, seguido de los bosques riparios $\left(\mathrm{H}^{\prime}=3.22\right)$. En la segunda campana los valores más altos se presentaron en los bancos forrajeros de leñosas $\left(\mathrm{H}^{\prime}=3.50\right)$, seguidos de los bosques riparios $\left(\mathrm{H}^{\prime}=3.47\right)$ y en la tercera campaña los valores más altos se presentaron en las pasturas naturales con alta densidad de árboles $\left(\mathrm{H}^{\prime}=3.32\right)$ y en los bosques riparios $\left(\mathrm{H}^{\prime}=3.32\right)$ con igual valor de diversidad. Como podemos observar, los bosques riparios se mantuvieron entre uno de los dos usos con los valores más altos durante las tres campañas de muestreo.

De igual forma al comparar los índices de diversidad (Shannon) entre los diferentes usos de suelos, sintetizando las tres campañas de muestreo, los bosques riparios encabezaron la 
lista de los tres valores más altos $\left(\mathrm{H}^{\prime}=3.92\right)$, seguido de los bosques secundarios intervenidos $\left(\mathrm{H}^{\prime}=3.86\right)$ y los bosques secundarios $\left(\mathrm{H}^{\prime}=3.80\right)$.

Cuadro 4. Índices ecológicos estructurales de aves calculados en 12 usos de suelo en los sistemas silvopastoriles de los municipios de Matiguás y Río Blanco y en el Cerro Quirragua (BPQ). Primera campaña.

\begin{tabular}{|l|c|c|c|c|c|c|}
\hline Usos & S & A & $\lambda$ & $H^{\prime}$ & E1 & E5 \\
\hline PNADA & 34 & 117 & 0.08 & 2.93 & 0.83 & 0.63 \\
\hline PMADA & 34 & 83 & 0.05 & 3.15 & 0.89 & 0.84 \\
\hline PNBDA & 36 & 96 & 0.04 & 3.35 & 0.94 & 1.00 \\
\hline PMBDA & 25 & 82 & 0.05 & 3.01 & 0.93 & 1.01 \\
\hline PNSA & 23 & 68 & 0.09 & 2.62 & 0.84 & 0.75 \\
\hline BFL & 33 & 132 & 0.07 & 3.01 & 0.86 & 0.86 \\
\hline CV & 31 & 84 & 0.06 & 3.05 & 0.90 & 0.81 \\
\hline TAC & 33 & 91 & 0.05 & 3.13 & 0.89 & 0.86 \\
\hline BR & 34 & 81 & 0.04 & 3.22 & 0.91 & 0.93 \\
\hline BSI & 32 & 80 & 0.04 & 3.18 & 0.92 & 0.97 \\
\hline BS & 16 & 24 & 0.05 & 2.61 & 0.94 & 1.48 \\
\hline BP & 14 & 26 & 0.05 & 2.52 & 0.95 & 1.59 \\
\hline BPQ & 12 & 34 & 0.12 & 2.17 & 0.87 & 0.93 \\
\hline
\end{tabular}

Cuadro 5. Índices ecológicos estructurales de aves calculados en 12 usos de suelo en los sistemas silvopastoriles de los municipios de Matiguás y Río Blanco y en el Cerro Quirragua (BPQ). Segunda campaña.

\begin{tabular}{|l|c|c|c|c|c|c|}
\hline UsOS & S & $\mathbf{A}$ & $\lambda$ & $\mathbf{H}^{\prime}$ & E1 & E5 \\
\hline PNADA & 42 & 140 & 0.04 & 3.35 & 0.90 & 0.88 \\
\hline PMADA & 32 & 143 & 0.05 & 3.12 & 0.90 & 0.88 \\
\hline PNBDA & 36 & 120 & 0.04 & 3.22 & 0.90 & 0.88 \\
\hline PMBDA & 37 & 115 & 0.05 & 3.21 & 0.89 & 0.71 \\
\hline PNSA & 20 & 63 & 0.07 & 2.68 & 0.89 & 0.95 \\
\hline BFL & 41 & 127 & 0.03 & 3.50 & 0.94 & 1.08 \\
\hline CV & 36 & 124 & 0.06 & 3.19 & 0.89 & 0.72 \\
\hline TAC & 35 & 124 & 0.08 & 2.97 & 0.83 & 0.61 \\
\hline BR & 49 & 165 & 0.04 & 3.47 & 0.89 & 0.78 \\
\hline BSI & 36 & 95 & 0.03 & 3.38 & 0.94 & 1.14 \\
\hline BS & 35 & 90 & 0.03 & 3.33 & 0.93 & 1.07 \\
\hline BP & 22 & 50 & 0.04 & 2.95 & 0.95 & 1.35 \\
\hline BPQ & 7 & 31 & 0.24 & 1.50 & 0.78 & 0.90 \\
\hline
\end{tabular}


Cuadro 6. Índices ecológicos estructurales de aves calculados en 12 usos de suelo en los sistemas silvopastoriles de los municipios de Matiguás y Río Blanco y en el Cerro Quirragua (BPQ). Tercera campaña.

\begin{tabular}{|l|c|c|c|c|c|c|}
\hline Usos & $\mathbf{S}$ & $\mathbf{A}$ & $\lambda$ & $\mathbf{H}^{\prime}$ & E1 & E5 \\
\hline PNADA & 39 & 131 & 0.04 & 3.32 & 0.91 & 0.88 \\
\hline PMADA & 32 & 76 & 0.09 & 2.88 & 0.83 & 0.61 \\
\hline PNBDA & 21 & 80 & 0.12 & 2.52 & 0.83 & 0.64 \\
\hline PMBDA & 17 & 127 & 0.14 & 2.30 & 0.81 & 0.70 \\
\hline PNSA & 8 & 40 & 0.21 & 1.71 & 0.82 & 0.83 \\
\hline BFL & 28 & 98 & 0.09 & 2.78 & 0.83 & 0.63 \\
\hline CV & 32 & 94 & 0.05 & 3.09 & 0.89 & 0.84 \\
\hline TAC & 34 & 140 & 0.05 & 3.17 & 0.90 & 0.87 \\
\hline BR & 27 & 152 & 0.10 & 2.71 & 0.82 & 0.60 \\
\hline BSI & 37 & 115 & 0.04 & 3.32 & 0.92 & 0.96 \\
\hline BS & 41 & 136 & 0.07 & 3.12 & 0.84 & 0.57 \\
\hline BP & 28 & 54 & 0.04 & 3.11 & 0.93 & 1.13 \\
\hline BPQ & 22 & 74 & 0.1 & 2.62 & 0.85 & 0.70 \\
\hline
\end{tabular}

Cuadro 7. Índices ecológicos estructurales de aves calculados en 12 usos de suelo en los sistemas silvopastoriles de los municipios de Matiguás y Río Blanco y en el Cerro Quirragua (BPQ). Síntesis de las tres campañas de monitoreo.

\begin{tabular}{|l|c|c|c|c|c|c|c|}
\hline Usos & S & A & $\lambda$ & H $^{\prime}$ & E1 & E5 & IB \\
\hline PNADA & 68 & 388 & 0.03 & 3.71 & 0.88 & 0.72 & 3.72 \\
\hline PMADA & 59 & 353 & 0.05 & 3.49 & 0.86 & 0.63 & 3.68 \\
\hline PNBDA & 62 & 296 & 0.03 & 3.65 & 0.88 & 0.73 & 3.53 \\
\hline PMBDA & 52 & 273 & 0.06 & 3.29 & 0.83 & 0.58 & 3.60 \\
\hline PNSA & 33 & 171 & 0.09 & 2.83 & 0.81 & 0.66 & 3.70 \\
\hline BFL & 59 & 357 & 0.04 & 3.57 & 0.88 & 0.73 & 3.76 \\
\hline CV & 60 & 302 & 0.03 & 3.65 & 0.89 & 0.77 & 3.65 \\
\hline TAC & 59 & 367 & 0.06 & 3.36 & 0.82 & 0.55 & 3.69 \\
\hline BR & 74 & 361 & 0.02 & 3.92 & 0.91 & 0.83 & 3.51 \\
\hline BSI & 72 & 311 & 0.03 & 3.86 & 0.90 & 0.75 & 3.67 \\
\hline BS & 63 & 168 & 0.03 & 3.80 & 0.92 & 0.84 & 3.70 \\
\hline BP & 41 & 150 & 0.04 & 3.34 & 0.90 & 0.83 & 3.78 \\
\hline BPQ & 22 & 87 & 0.07 & 2.74 & 0.89 & 0.88 & 3.52 \\
\hline
\end{tabular}

Las abundancias entre las campañas de muestreo no mostraron diferencias significativas $(\mathrm{X} 2 \mathrm{f}=4.30, \mathrm{p}=0.11)$. Los valores de abundancia entre los usos mostraron diferencias muy significativas $(H=38, p<0.01)$ (ver ilustración 10).Los usos en los que se encontró una mayor abundancia de individuos considerando todas las campañas fueron las pasturas naturales con alta densidad de árboles y los tacotales. 


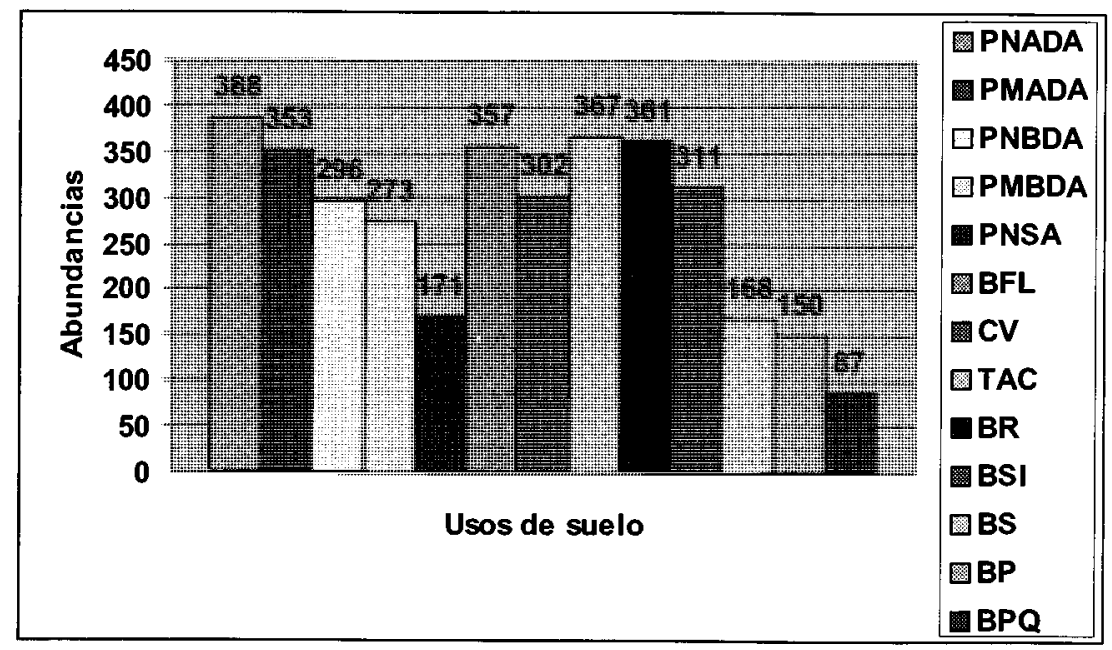

llustración 10. Abundancias totales por usos de suelo

150 Entre las riquezas de especies entre campañas no se encontraron diferencias significativas $(\mathrm{X} 2 \mathrm{f}=3.16, \mathrm{p}=0.2)$. Los valores de riqueza entre los usos mostraron diferencias muy significativas $(H=45.19, \mathrm{p}<0.01)$. Los usos con mayor riqueza fueron los bosques riparios y los bosques secundarios intervenidos (ver cuadro 7 e ilustración 11), que a su vez fueron los usos con valores más altos de los índices de diversidad.

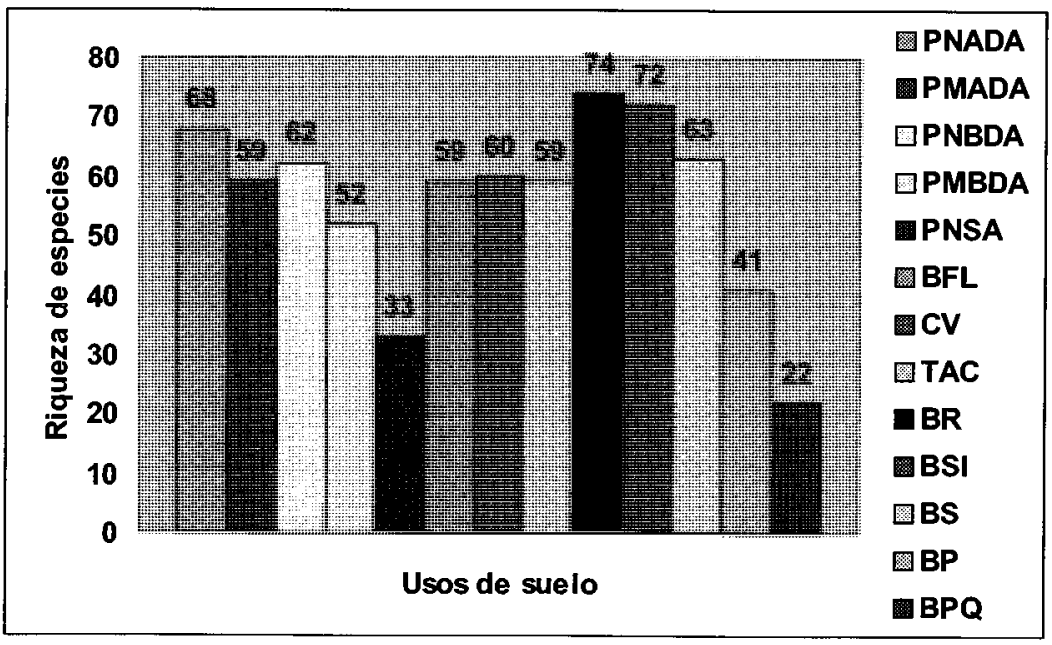

Ilustración 11. Riquezas de especies por usos de suelo

Nicho ecológico: Los datos sobre el nicho ecológico se presentan en el cuadro 8. En relación con la dependencia del bosque, no se observó ninguna especie estrictamente dependiente del mismo, un $2.65 \%$ de especies con dependencia entre media y ninguna $(1,2)$, un $39 \%$ de especies con dependencia media (2), un $35 \%$ de especies con dependencia entre media y alta $(2,3)$, un $36.4 \%$ de especies no dependientes del bosque (3) (ver ilustración 12). 
Cuadro 8. Gremios alimenticios y dependencia del bosque

\begin{tabular}{|c|c|c|c|}
\hline Nombre científico & Estado & $\begin{array}{c}\text { Recurso } \\
\text { alimenticio }\end{array}$ & $\begin{array}{c}\text { Dependencia } \\
\text { del bosque }\end{array}$ \\
\hline Bubulcus ibis & $\mathrm{R}, \mathrm{M}$ & Artrópodos & 3 \\
\hline Mycteria americana & $\mathrm{R}$ & Vertebrados & 3 \\
\hline Coragyps atratus & $\mathrm{R}$ & Carroña & 3 \\
\hline Cathartes aura & $\mathrm{R}, \mathrm{M}$ & Carroña & 3 \\
\hline Elanoides forficatus & $S$ & Vertebrados & 2 \\
\hline Asturina nitida & $\mathrm{R}$ & Vertebrados & 2 \\
\hline Caracara plancus & $\mathrm{R}$ & Carroña & 3 \\
\hline Herpetotheres cachinna & $\mathrm{R}$ & Vertebrados & 2 \\
\hline Ortalis cinereiceps & $\mathrm{R}$ & Frutas & 2,3 \\
\hline Columbina inca & $\mathrm{R}$ & Semillas & 3 \\
\hline Columbina minuta & $\mathrm{R}$ & Semillas & 3 \\
\hline Columbina talpacoti & $\mathrm{R}$ & Semillas & 3 \\
\hline Columba flavirostris & $\mathrm{R}$ & Semillas & 3 \\
\hline Leptotila cassinii & $\mathrm{R}$ & Semillas & 2,3 \\
\hline Leptotila verreauxi & $\mathrm{R}$ & Semillas & 2,3 \\
\hline Aratinga nana & $\mathrm{R}$ & Frutas & 2,3 \\
\hline Brotogeris jugularis & $\mathrm{R}$ & $\begin{array}{l}\text { Frutas, } \\
\text { Semillas }\end{array}$ & 3 \\
\hline Amazona albifrons & $\mathrm{R}$ & Semillas & 2 \\
\hline Amazona autumnalis & $\mathrm{R}$ & $\begin{array}{l}\text { Frutas, } \\
\text { Semillas }\end{array}$ & 2 \\
\hline Piaya cayana & $\mathrm{R}$ & Artrópodos & 2,3 \\
\hline Tapera naevia & $\mathrm{R}$ & Artrópodos & 3 \\
\hline Morococcys erythropygus & $\mathrm{R}$ & Artrópodos & 2,3 \\
\hline Crotophaga sulcirostris & $\mathrm{R}$ & Artrópodos & 3 \\
\hline Tyto alba & $\mathrm{R}$ & Vertebrados & 2 \\
\hline Otus cooperi & $\mathrm{R}$ & Artrópodos & 2 \\
\hline Pseudoscops clamator & $\mathrm{R}$ & Vertebrados & 2 \\
\hline Nyctibius griseus & $\mathrm{R}$ & Artrópodos & 2,3 \\
\hline Caprimulgus vociferus & M & Artrópodos & 2,3 \\
\hline Chordeiles acutipennis & $\mathrm{R}$ & Artrópodos & 3 \\
\hline Amazilia rutila & $\mathrm{R}$ & Nectar & 2,3 \\
\hline A mazilia tzacatl & $\mathrm{R}$ & Nectar & 3 \\
\hline Amazilia cyanura & $\mathrm{R}$ & Nectar & 2,3 \\
\hline Amazilia saucerrottei & $\mathrm{R}$ & Nectar & 2,3 \\
\hline Archilocus colubris & $\mathrm{M}$ & Nectar & 3 \\
\hline Eupherusa eximia & $\mathrm{R}$ & Nectar & 2 \\
\hline Phaethornis longuemareus & $\mathrm{R}$ & Nectar & 2,3 \\
\hline Phaethornis superciliosus & $\mathrm{R}$ & Nectar & 2 \\
\hline Anthracothorax prevostii & $\mathrm{R}$ & Nectar & 3 \\
\hline
\end{tabular}




\begin{tabular}{|c|c|c|c|}
\hline Trogon melanocephalus & $\mathrm{R}$ & Frutas & 2 \\
\hline Trogon violaceus & $\mathrm{R}$ & Frutas, Insectos & 2,3 \\
\hline Chloroceryle americana & $\mathrm{R}$ & Vertebrados & 2,3 \\
\hline Pteroglossus torquatus & $\mathrm{R}$ & Omnivoro & 2 \\
\hline Ramphastos sulfuratus & $R$ & Omnivoro & 2 \\
\hline Melanerpes hoffmannii & $\mathrm{R}$ & Frutas, insectos & 2,3 \\
\hline Piculus rubiginosus & $R$ & Artropodos & 2 \\
\hline Dryocopus lineatus & $\mathrm{R}$ & Artrópodos & 2,3 \\
\hline Sittasomus griseicapillus & $\mathrm{R}$ & Artrópodos & 2 \\
\hline Lepidocolaptes souleyetii & $\mathrm{R}$ & Artrópodos & 2,3 \\
\hline Dendrocinchla homocroa & $\mathrm{R}$ & Artrópodos & 1,2 \\
\hline Thamnophilus doliatus & $\mathrm{R}$ & Artrópodos & 2,3 \\
\hline Cercomacra tyrannina & $\mathrm{R}$ & Artrópodos & 2,3 \\
\hline Myrmornis torquata & $\mathrm{R}$ & Artrópodos & 1,2 \\
\hline Camptostoma imberbe & $R$ & Frutas, Insectos & 2 \\
\hline Camptostoma obsoletum & $\mathrm{R}$ & Frutas, Insectos & 2,3 \\
\hline Mionectes oleagineus & $\mathrm{R}$ & Artrópodos & 2 \\
\hline Elaenia flavogaster & $\mathrm{R}$ & Artrópodos & 3 \\
\hline Todirostrum cinereum & $\mathrm{R}$ & Artr ópodos & 2,3 \\
\hline Tolmomyias sulphurescens & $\mathrm{R}$ & Artrópodos & 3 \\
\hline Contopus cinereus & $\mathrm{R}$ & Artrópodos & 3 \\
\hline Contopus virens & $M$ & Artrópodos & 2,3 \\
\hline Empidonax albigularis & $\mathrm{R}$ & Artrópodos & 2,3 \\
\hline Empidonax flaviventris & $M$ & Artrópodos & 2 \\
\hline Empidonax flavescens & $\mathrm{R}$ & Artrópodos & 2 \\
\hline Empidonax minimus & $M$ & Artrópodos & 2,3 \\
\hline Attila spadiceus & $\mathrm{R}$ & Artrópodos & 2 \\
\hline Myiarchus tuberculifer & $\mathrm{R}$ & Artrópodos & 2,3 \\
\hline Myiarchus tyranulus & $\mathrm{R}$ & Artrópodos & 2,3 \\
\hline Myiarchus cinerascens & $M$ & Artrópodos & 2,3 \\
\hline Myiarchus nuttingi & $\mathrm{R}$ & Artrópodos & 2 \\
\hline Myiarchus crinitus & $\mathrm{M}$ & Artrópodos & 2 \\
\hline Pitangus s ulfuratus & $\mathrm{R}$ & Artrópodos & 3 \\
\hline Megarhynchus pitangua & $\mathrm{R}$ & Artrópodos & 2,3 \\
\hline Myiozetetes similis & $\mathrm{R}$ & Frutas, insectos & 3 \\
\hline Myiodynastes luteiventris & $\mathrm{M}$ & Artrópodos & 2,3 \\
\hline Tyrannus melancholicus & $\mathrm{R}$ & Artrópodos & 3 \\
\hline Tyrannus forficatus & $\mathrm{M}$ & Frutas, Insectos & 3 \\
\hline Tyrannus savana & $\mathrm{R}$ & Insectos, Frutos & 3 \\
\hline Pachyramphus polychopterus & $\mathrm{R}$ & Frutas, Insectos & 2,3 \\
\hline Tityra semifasciata & $\mathrm{R}$ & Frutas, Insectos & 2,3 \\
\hline
\end{tabular}




\begin{tabular}{|c|c|c|c|}
\hline Chiroxiphia linearis & $\mathrm{R}$ & Frutas & 2 \\
\hline Manacus candei & $\mathrm{R}$ & Frutas & 2 \\
\hline Cyclarhis gujanensis & $\mathrm{R}$ & Artrópodos & 2,3 \\
\hline Vireo flavifrons & $\mathrm{R}, \mathrm{M}$ & Artrópodos & 2 \\
\hline Vireo flavoviridis & $\mathrm{R}$ & Artrópodos & 3 \\
\hline Vireo olivaceus & $P$ & Artrópodos & 3 \\
\hline Hylophilus decurtatus & $\mathrm{R}$ & Artrópodos & 2,3 \\
\hline Calocitta formosa & $\mathrm{R}$ & Omnivoro & 2,3 \\
\hline Cyanocorax morio & $\mathrm{R}$ & Omnivoro & 3 \\
\hline Hirundo rustica & $\mathrm{P}$ & Insectos, Frutos & 3 \\
\hline Procnes chalybea & $\mathrm{R}, \mathrm{M}$ & Insectos, Frutos & 3 \\
\hline Campylorhynchus rufinucha & $\mathrm{R}$ & Artrópodos & 2,3 \\
\hline Thryothorus rufalbus & $\mathrm{R}$ & Artrópodos & 1,2 \\
\hline Thryothorus modestus & $\mathrm{R}$ & Artrópodos & 3 \\
\hline Thryothorus maculipectus & $\mathrm{R}$ & Artrópodos & 3 \\
\hline Troglodytes aedon & $\mathrm{R}$ & Artrópodos & 3 \\
\hline Polioptila albiloris & $\mathrm{R}$ & Artrópodos & 2,3 \\
\hline Ramphocaenus melanurus & $\mathrm{R}$ & Artrópodos & 2,3 \\
\hline Hylocichla mustelina & $M$ & Artrópodos & 1,2 \\
\hline Turdus grayi & $\mathrm{R}$ & Frutas, Insectos & 3 \\
\hline Turdus assimilis & $\mathrm{R}$ & Frutas, Insectos & 2 \\
\hline Catharus aurantirostris & $\mathrm{R}$ & Artrópodos & 2,3 \\
\hline Vermivora peregrina & $M$ & Artrópodos & 2,3 \\
\hline Vermivora chryspotera & $M$ & Artrópodos & 2 \\
\hline Parula pitiayumi & $\mathrm{R}$ & Artrópodos & 2 \\
\hline Dendroica petechia & $\mathrm{R}, \mathrm{M}$ & Artrópodos & 3 \\
\hline Dendroica pensylvanica & $M$ & Artrópodos & 2,3 \\
\hline Dendroica magnolia & M & Artrópodos & 2,3 \\
\hline Dendroica fusca & $M$ & Artrópodos & 2 \\
\hline Dendroica castanea & M & Artrópodos & 2,3 \\
\hline Dendroica towsendi & $M$ & Artrópodos & 2,3 \\
\hline Dendroica discolor & $M$ & Artrópodos & 2,3 \\
\hline Mniotilta varia & $M$ & Artrópodos & 2 \\
\hline Setophaga ruticilla & M & Artrópodos & 2,3 \\
\hline Seiurus aurocapillus & $M$ & Artrópodos & 2 \\
\hline Geothlypis poliocephala & $\mathrm{R}$ & Artrópodos & 3 \\
\hline Geothlypis aequinoctialis & $\mathrm{R}$ & Artrópodos & 3 \\
\hline Wilsonia citrina & $\mathrm{M}$ & Artrópodos & 2 \\
\hline Wilsonia canadensis & $M$ & Artrópodos & 2,3 \\
\hline Basileuterus rufifrons & $\mathrm{R}$ & Artrópodos & 2 \\
\hline Habia fuscicauda & $\mathrm{R}$ & Frutos, Insectos & 2 \\
\hline
\end{tabular}


154

\begin{tabular}{|c|c|c|c|}
\hline Piranga rubra & $M$ & Frutos, Insectos & 2,3 \\
\hline Piranga olivacea & $P$ & Frutos, Insectos & 3 \\
\hline Ramphocelus sanguinolentus & $\mathrm{R}$ & Frutos, Insectos & 3 \\
\hline Ramph ocelus passerinii & $\mathrm{R}$ & Frutos, insectos & 3 \\
\hline Thraupis episcopus & $\mathrm{R}$ & Frutos & 3 \\
\hline Thraupis abbas & $R$ & Frutos & 3 \\
\hline Tangara lavinia & $\mathrm{R}$ & Frutos, Insectos & 2 \\
\hline Tangara larvata & $\mathrm{R}$ & Frutos, Insectos & 3 \\
\hline Euphonia hirundinacea & $R$ & Frutas & 2,3 \\
\hline Euphjonia affinis & $R$ & Frutas & 2 \\
\hline Euphonia luteicapilla & $\mathrm{R}$ & Frutas & 2,3 \\
\hline Euphonia gouldi & $\mathrm{R}$ & Frutas & 2 \\
\hline Cyanerpes cyaneus & $\mathrm{R}$ & Semillas & 2 \\
\hline Volatinia jacarina & $\mathrm{R}$ & Semillas & 3 \\
\hline Sporophila torqueola & $\mathrm{R}$ & Semillas & 3 \\
\hline Sporophila aurita & $\mathrm{R}$ & Semillas & 3 \\
\hline Melozone leucotis & $\mathrm{R}$ & Artrópodos & 2,3 \\
\hline Tiaris olivacea & $\mathrm{R}$ & Semillas & 3 \\
\hline Arrem onops conirostris & $\mathrm{R}$ & Artrópodos & 2,3 \\
\hline Oryzoborus funereus & $\mathrm{R}$ & Semillas & 3 \\
\hline Saltator maximus & $\mathrm{R}$ & Frutos, Insectos & 3 \\
\hline Saltator coerulescens & $\mathrm{R}$ & Frutos, insectos & 3 \\
\hline Caryothraustes poliogaster & $\mathrm{R}$ & Artrópodos & 2 \\
\hline Sturnella magna & $\mathrm{R}, \mathrm{M}$ & Artrópodos & 3 \\
\hline Icterus spurius & M & Frutas & 3 \\
\hline Icterus galbula & $M$ & Frutas, Insectos & 2,3 \\
\hline Icterus dominicensis & $\mathrm{R}$ & $\begin{array}{c}\text { Artrópodos, } \\
\text { Frutos }\end{array}$ & 3 \\
\hline Dives dives & $\mathrm{R}$ & Artrópodos & 3 \\
\hline Amblycercus holosericeus & $\mathbf{R}$ & Artrópodos & 3 \\
\hline Quiscalus mexicanus & $\mathrm{R}$ & Omnivoro & 3 \\
\hline Psaracolius montezuma & $\mathrm{R}$ & Frutas, Insectos & 2,3 \\
\hline
\end{tabular}




\section{Tipos dependencia del bosque}

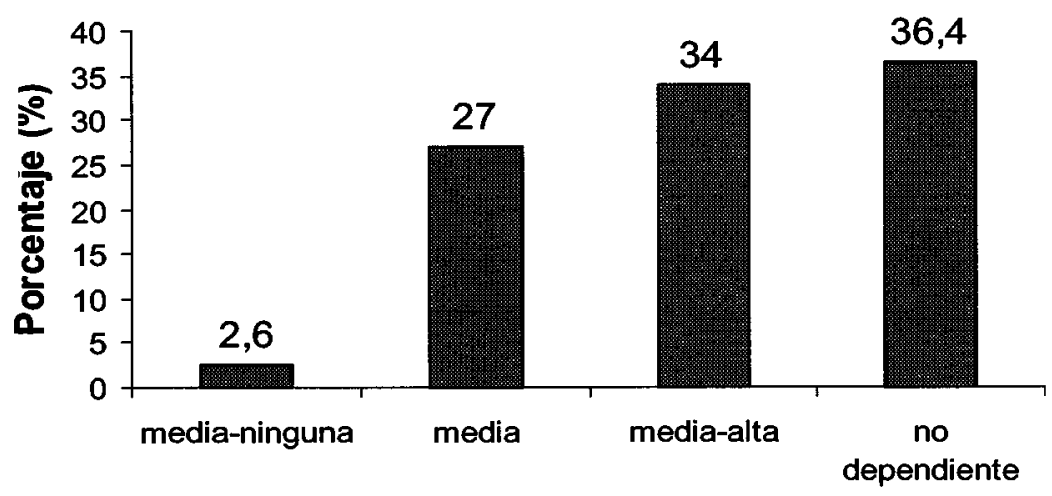

llustración 12. Dependencia del bosque

En relación con los gremios alimenticios (ver ilustración 13), se observó un 50 \% de especies insectívoras, un $17 \%$ de insectívoras-frugívoras, $8.7 \%$ granívoras, $8 \%$ frugívoras, $6 \%$ nectarívoras y un $3.33 \%$ de especies omnívoras.

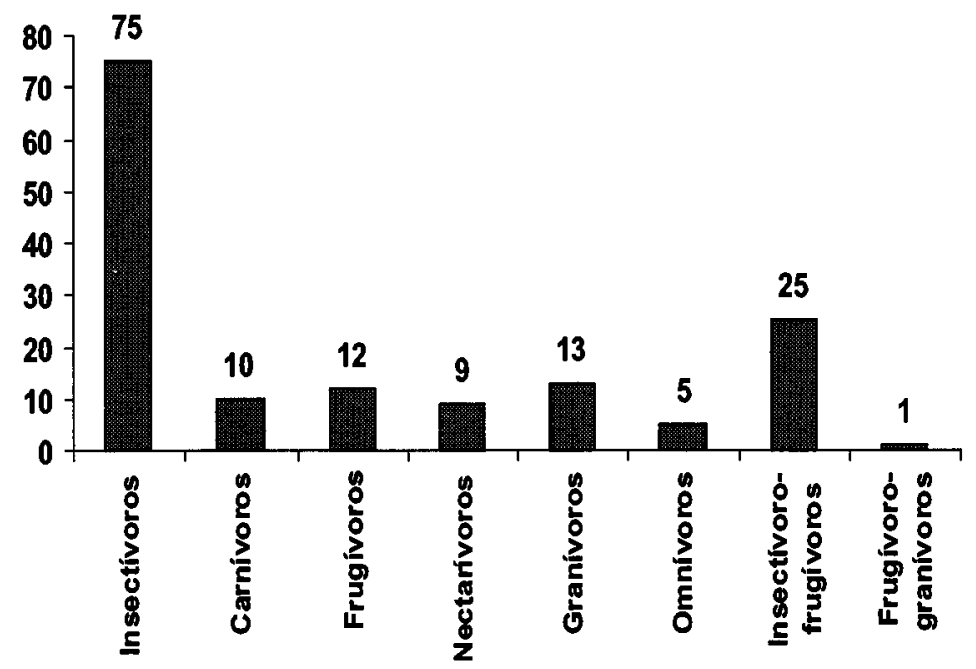

llustración 13. Sintesis de la información sobre gremios alimentarios

Análisis de agrupamiento: El análisis de agrupamiento (ver ilustración 14) muestra la formación de dos grupos, uno compuesto por las especies del bosque primario de Quirragua (BPP) y el otro por todas las comunidades estudiadas en los sistemas silvopastoriles. Dentro de estas últimas se puede observar a su vez la conformación de tres grupos; uno formado por las PNSA, el otro conformado por los usos boscosos y las pasturas mejoradas con baja densidad de árboles y el tercero, conformado por los usos de pasturas, banco forrajero de leñosas, tacotales y cercas vivas. 


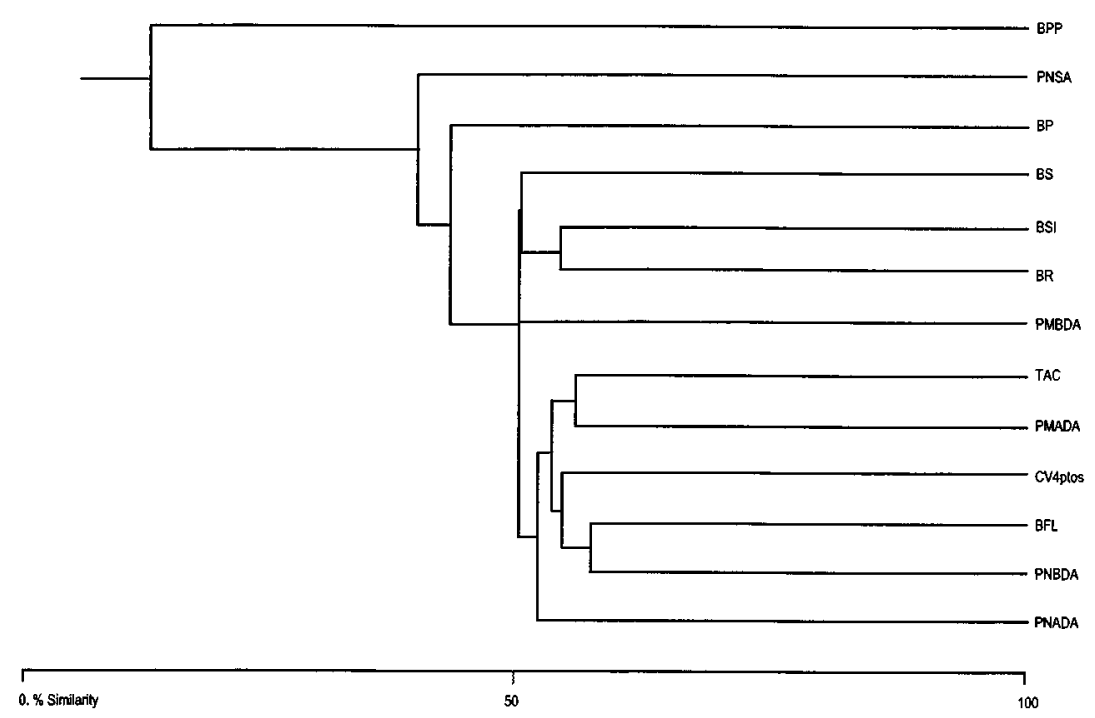

156 Ilustración 14. Dendrograma de similitud de los tipos de uso en relación con la presencia o ausencia de especies de aves

\section{Moluscos:}

Se observó un total de 51 especies distribuidas en 20 familias y tres órdenes. Las familias mejor representadas en la zona fueron Subulinidae y Spiraxidae (ver ilustración 15).

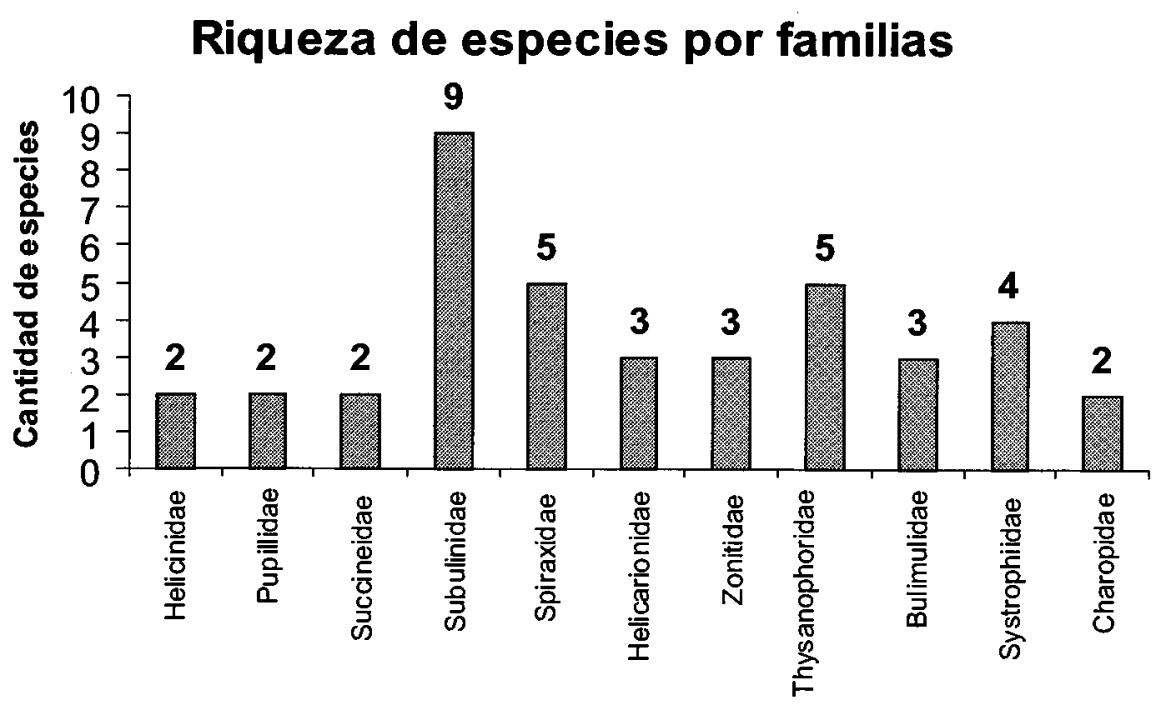

llustración 15. Cantidad de especies por familias

Estructura de las comunidades: Al igual que en los otros grupos estudiados, las curvas de saturación de especies según los índices de Chao1 y Chao2 muestran un comportamiento que todavía no es asintótico, lo que significa que existen nuevas especies a encontrar en la zona (ver ilustración 16); el índice de Chao1 predice 53 especies de moluscos y el índice de Chao2 64, valores que no están muy alejados de los encontrados en el campo. 


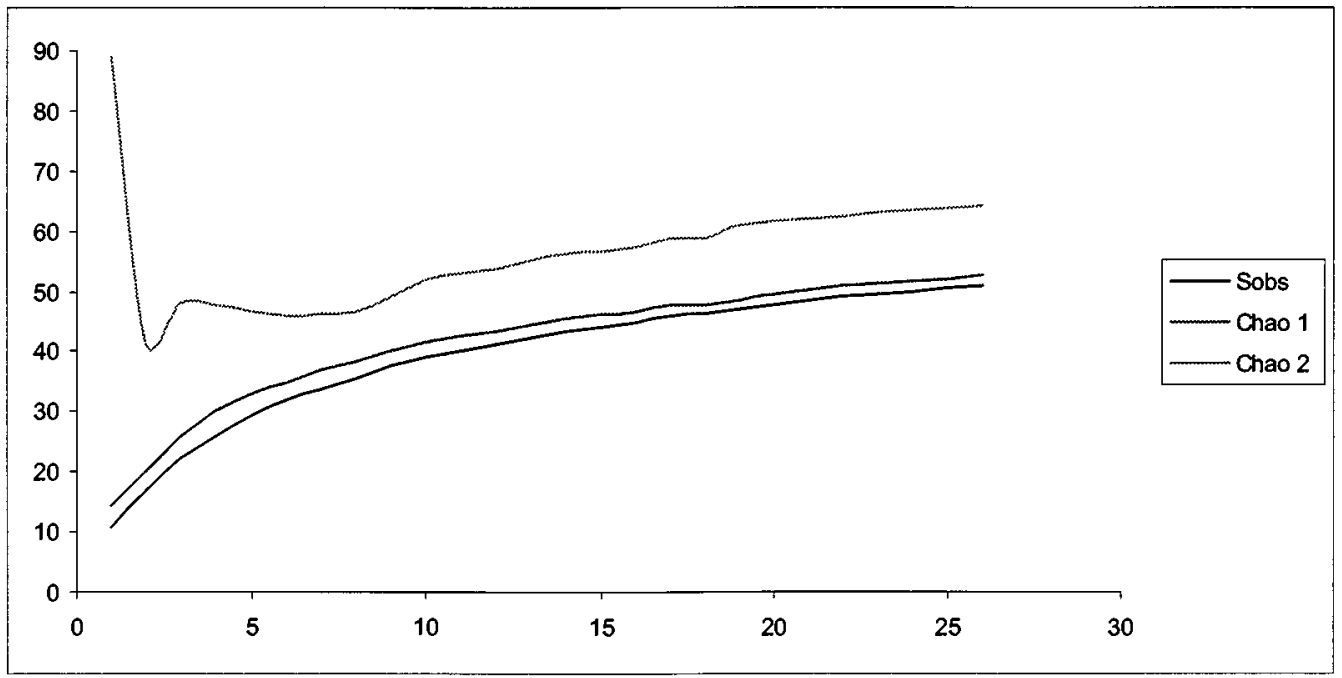

Ilustración 16. Curva de saturación de especies de moluscos

De los usos estudiados, el más rico resultó el bosque primario, seguido del bosque ripario (ver cuadro 9), aunque estos resultados podrían modificarse cuando termine de procesarse el material recolectado.

Cuadro 9. Riqueza de especies de moluscos calculados en 12 usos de suelo de los sistemas silvopastoriles de los municipios de Matiguás y Río Blanco y en el Cerro Quirragua (BPQ).

\begin{tabular}{|c|c|c|c|c|c|c|c|}
\hline Indices & $\mathbf{S}$ & $\mathbf{A}$ & $\mathbf{H}^{\prime}$ & $\lambda$ & $\mathbf{E 1}$ & $\mathbf{E 5}$ & $\mathbf{I B}$ \\
\hline PNBDA & 13 & 189 & 1.62 & 0.33 & 0.63 & 0.51 & 3.31 \\
\hline PNADA & 6 & 88 & 0.75 & 0.66 & 0.42 & 0.46 & 2.83 \\
\hline PMADA & 13 & 208 & 1.38 & 0.42 & 0.54 & 0.47 & 3.00 \\
\hline PMBDA & 8 & 95 & 1.17 & 0.48 & 0.56 & 0.48 & 2.88 \\
\hline PNSA & 9 & 94 & 1.15 & 0.4 & 0.52 & 0.7 & 3.22 \\
\hline C.V. & 7 & 75 & 1.39 & 0.33 & 0.71 & 0.68 & 2.71 \\
\hline BFL & 18 & 149 & 2.24 & 0.13 & 0.77 & 0.77 & 2.94 \\
\hline TAC & 21 & 299 & 2.13 & 0.18 & 0.7 & 0.61 & 2.90 \\
\hline BS & 21 & 150 & 2.04 & 0.25 & 0.67 & 0.44 & 3.29 \\
\hline BSI & 15 & 251 & 1.49 & 0.43 & 0.55 & 0.39 & 3.40 \\
\hline BR & 23 & 153 & 2.2 & 0.19 & 0.7 & 0.51 & 3.09 \\
\hline BP & 31 & 1,040 & 2.87 & 0.07 & 0.84 & 0.74 & 3.32 \\
\hline BPQ & 17 & 91 & 2.22 & 0.15 & 0.79 & 0.68 & 4.13 \\
\hline
\end{tabular}

Análisis de agrupamiento: $\mathrm{El}$ análisis de agrupamiento (ver ilustración 17) muestra la formación de dos grupos, uno formado por el bosque primario de Quirragua y otro por todos los usos en sistemas silvopastoriles. Dentro de los usos relacionados con sistemas se forman dos grupos más o menos definidos: uno compuesto por BSI, PMBDA, PNADA y cercas vivas y un segundo grupo compuesto por todos los otros usos. 


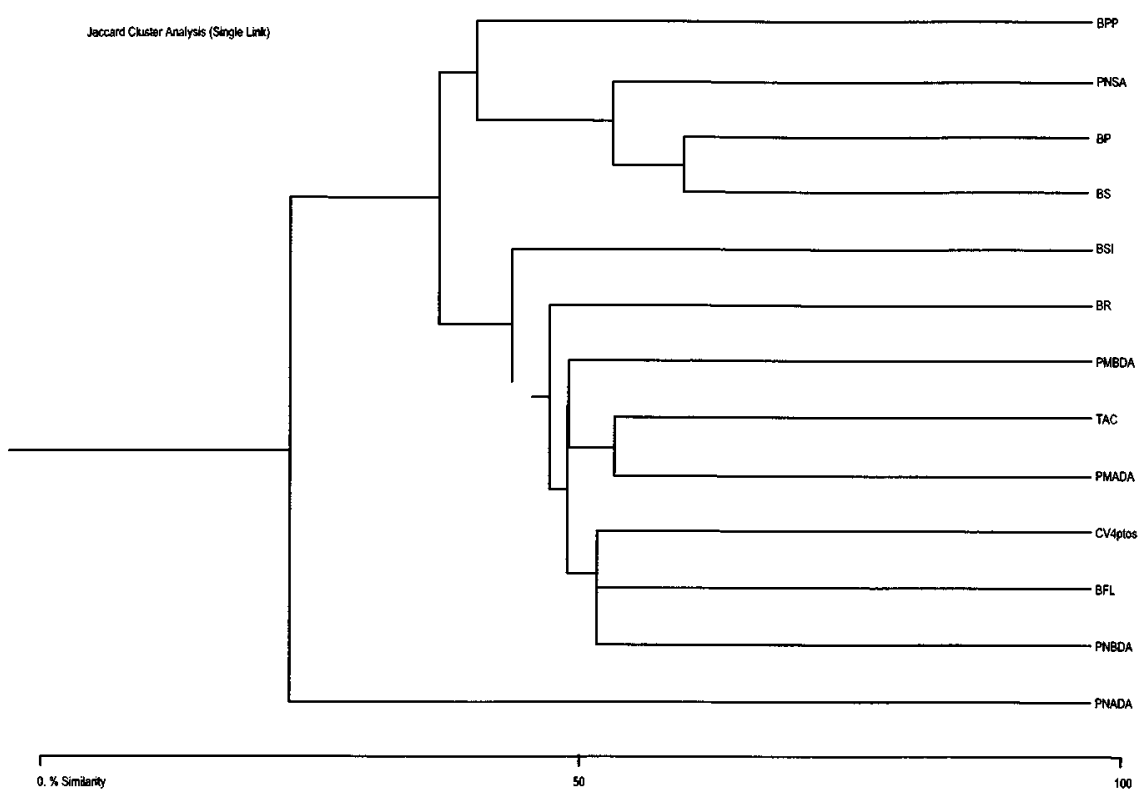

llustración 17. Dendrograma de similitud de los tipos de uso en relación con la presencia o ausencia de especies de moluscos.

\section{Discusión}

Análisis general: En 1983, en el país se estimó una cobertura total de bosques de 76,668 km2. En el año 2000, la cobertura de bosques del país se estima en 55,977 km2. De estos datos se calcula una pérdida total de bosques en el país de 20,691 km2, transformada en su mayoría en tierras bajo uso agropecuario y asentamientos. Eso quiere decir que en 17 años, Nicaragua perdió el 27 \% del bosque que tenía, lo que representa una tasa anual de conversión de uso del suelo del $1.6 \%$ (MARENA, 2001).

El desarrollo de las actividades agropecuarias es la causa principal del avance de la frontera agrícola y ha provocado la creación de pequeños parches o islotes de bosques dispersos en diferentes zonas, es decir, la fragmentación forestal. De acuerdo a Murcia (1995), la fragmentación del bosque es el reemplazo de grandes áreas del bosque nativo por otros ecosistemas, dejando parches o islas separados de bosque, con consecuencias deletéreas para la biota nativa. Esta fragmentación tiene dos componentes principales:

* Reducción y pérdida de la cantidad total del tipo de hábitat e incluso de todo hábitat natural en un paisaje.

* Separación del hábitat remanente en parches más pequeños y aislados.

Ambos contribuyen a la disminución progresiva de la diversidad biológica (Harris, 1984). Wilcox (1980) señala que a medida que la fragmentación del bosque procede, el tamaño de los fragmentos disminuye y el aislamiento aumenta, conformándose los llamados "hábitats islas". Esto facilitaría la extinción o exterminación total de una o más especies y la preservación diferenciada de otras (Harris, 1984). 
Los datos previos existentes de la cuenca de Matiguás (Morales et al., 2002) (ver ilustración 18), muestran una clara regresión de la cobertura en el área entre 1981 y 1987. En este sentido, es de gran importancia tener en cuenta que según Pérez et al. (2003), la variable más importante relacionada con la biodiversidad en los sistemas silvopastoriles es la cobertura.

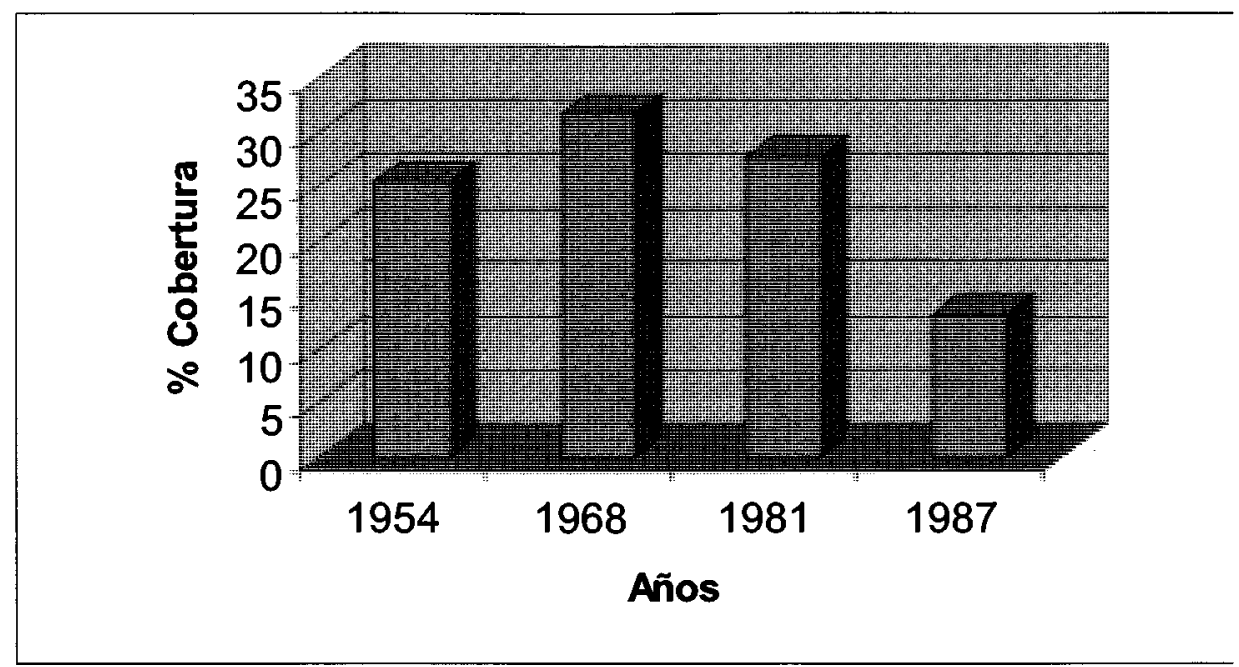

llustración 18. Variación del porcentaje de cobertura en la cuenca de Bulbul (según Morales et al. 2002).

Vegetación: La vegetación constituye el elemento más importante en la mayoría de los ecosistemas de todos los medios, terrestre, marino y fluviátil, hasta el punto que constituye el $99 \%$ de biomasa de la tierra (vid. Pérez, 2001). La diversidad florística de Nicaragua es de 6,500 especies según WCMC (1992), lo que constituye un $2.7 \%$ de la diversidad florística global.

En la cuenca de Bulbul, en Matiguás se han identificado hasta el presente un total de 170 especies de plantas distribuidas en 57 familias, teniendo en cuenta las especies procedentes de los sistemas silvopastoriles estudiados, tanto de las parcelas de estudio como las más notables encontradas fuera de las parcelas, así como las especies recolectadas en el Cerro Quirragua.

En general, en los usos de suelo muestreados en la cuenca de Bulbul se contabilizaron 1,896 individuos. Basándonos en la escala cualitativa de abundancia de Tansley y Chipp (1926), fueron identificadas como especies muy abundantes: Bursera simarouba (Burseraceae), abundantes: Guazuma ulmifolia (Sterculiaceae), Poco Abundantes: Cordia alliodora (Boraginaceae), escasas: Tabebuia rosea (Bignoniaceae), Gliricidia sepium (Fabaceae), Trichilia sp. (Meliaceae) y Raras: Liquidambar styraciflua (Hamamelidaceae), Ardisia revoluta (Myrcinaceae) Platymiscium pleiostachyum (Fabaceae), Albizia caribea y todas las demás especies identificadas.

En cuanto a la riqueza de especies, los valores más altos obtenidos se encontraron en el bosque primario del Cerro Quirragua (47) y en los bosques riparios (43), que son los dos usos de suelo más conservados en el área de estudio. Los valores más altos de diversidad fueron 
obtenidos en bosque ripario $\left(\mathrm{H}^{\prime}=2.63\right)$, bosque secundario intervenido $\left(\mathrm{H}^{\prime}=2.50\right)$ y el tacotal $\left(\mathrm{H}^{\prime}=2.38\right)$, y los valores más bajos se encontraron en pastura mejorada con baja densidad de árboles $\left(\mathrm{H}^{\prime}=1.12\right)$, cerca viva $\left(\mathrm{H}^{\prime}=1.49\right)$ y banco forrajero de leñosas $\left(\mathrm{H}^{\prime}=1.81\right)$. Los valores de equitatividad más altos fueron obtenidos en el bosque secundario intervenido $(\mathrm{E} 1=0.79$ y $\mathrm{E} 5=0.62)$ y tacotal $(\mathrm{E} 1=0.76$ y $\mathrm{E} 5=0.62)$.

Aves: Las aves son el grupo más biodiverso de vertebrados en el nivel global y, posiblemente, el más utilizado para el monitoreo de la biodiversidad. Existen unas 9,672 especies conocidas en el nivel global y 676 para Nicaragua según WMCM (1992), lo que representa un 6.98 \% del total global.

En la cuenca de Matiguás se han identificado hasta el presente 170 especies de aves, que representa una cifra alta a nuestro juicio. En general, en los usos de suelo muestreados en las cuencas de Bulbul y Paiwas se censó un total de 3,575 individuos. Las especies más abundantes fueron Volatinia jacarina (308), Crotophaga sulcirostris (283), Troglodytes aedon (162) y Tyrannus melancholicus (121).

Moluscos: El filo Molusco constituye uno de los grupos faunísticos más diversos después de los artrópodos, con unas 130,000 especies descritas (Remane et al., 1980) de las cuales unas 60,000 son especies de gasterópodos o caracoles (Solem, 1974). Este grupo se distribuye en el mar, en aguas dulces y en la tierra. Según Pérez et al. (2003) en Nicaragua existen unas 1,900 especies de moluscos, de las cuales los gasterópodos son ca. 1,436 lo que constituye aproximadamente un $2.4 \%$ de ese total.

En la cuencas de Bulbul y Paiwas se han identificado hasta el presente 35 especies de moluscos, lo cual es lo esperado en comunidades de moluscos como plantean Solem y Climo (1985), Pérez et al., (1996) y Pérez et al. (en prensa) entre otros autores.

En general, en los usos de suelo muestreados en las cuencas de Bulbul y Paiwas, se contabilizaron 2,882 individuos. Las especies más abundantes fueron Bulimulus corneus (732 individuos), Caecilioides consobrinus (280) y Lucidella lirata (220).

Relaciones estructurales: Se calcularon valores de diversidad estructural y de diversidad ecológica para cada una de las parcelas estudiadas y se realizó una prueba de correlación de Pearson para determinar si existe una relación entre la heterogeneidad de la estructura de la vegetación y la diversidad de especies.

Los resultados indican que existe una muy significativa correlación entre la diversidad ecológica y la diversidad estructural $(\mathrm{r}=0.344, \mathrm{P}<0.01)$ (ver ilustración 19), aumentando la diversidad estructural con un incremento de la diversidad ecológica. 


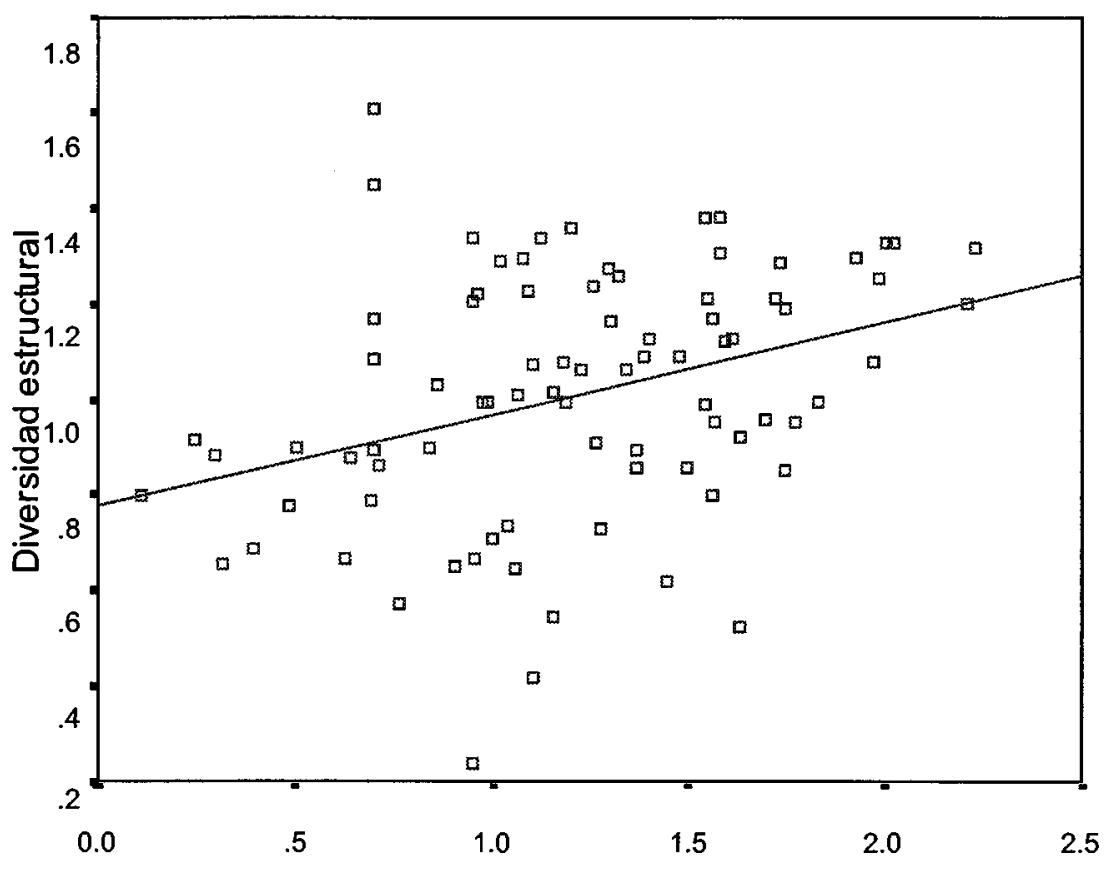

Diversidad ecológica

Ilustración 19. Relación entre la diversidad de especies o diversidad ecológica y la diversidad estructural o complejidad del follaje

Estos resultados son de gran importancia, ya que si la diversidad estructural está correlacionada de manera altamente significativa con la diversidad de especies, la "buena salud" de los ecosistemas vegetales no puede ser evaluada teniendo en cuenta sólo los datos de cobertura como se hace habitualmente. Este planteamiento ya fue realizado por Pérez et al., (2004) en un trabajo anterior, de manera preliminar.

\section{Conclusiones}

1. Se encontró un total de 180 especies de aves, de ellas 151 fueron contabilizadas en los puntos de conteo.

2. De las 151 especies contabilizadas en las parcelas de muestreo, 113 son especies residentes y 38 son especies migratorias.

3. La cantidad de especies de aves por campañas de muestreo y en total, se presentan en el siguiente cuadro. En el mismo se puede apreciar que la cantidad de especies fue mayor durante la segunda campaña.

\begin{tabular}{|c|c|c|c|c|}
\hline & $\begin{array}{c}\text { Primera } \\
\text { campaña }\end{array}$ & $\begin{array}{c}\text { Segunda } \\
\text { campaña }\end{array}$ & $\begin{array}{c}\text { Tercera } \\
\text { campaña }\end{array}$ & $\begin{array}{c}\text { Total } \\
\text { (Tres } \\
\text { campañas) }\end{array}$ \\
\hline Riqueza & 98 & 107 & 94 & 151 \\
\hline
\end{tabular}


4. Los valores más altos de riqueza de especies en plantas, se encontraron en el bosque primario del Cerro Quirragua $(S=46)$ y en los bosques riparios $(S=43)$; en aves se encontraron en los bosques riparios $(S=74)$ y en bosques secundarios intervenidos $(S=72)$; y en moluscos en bosques primarios $(S=31)$, seguidos por los bosques riparios $(S=23)$.

5. En cuanto a la diversidad, los valores más altos para plantas se encontraron en el bosque primario de Quirragua $\left(\mathrm{H}^{\prime}=3.34\right)$ y en los bosques riparios $\left(\mathrm{H}^{\prime}=3.12\right)$; para aves se encontraron en bosques riparios $\left(\mathrm{H}^{\prime}=3.92\right)$ y bosques secundarios intervenidos $\left(\mathrm{H}^{\prime}=3.86\right)$; y en moluscos, en bosques primarios $\left(\mathrm{H}^{\prime}=2.87\right)$ y en bancos forrajeros de leñosas $\left(H^{\prime}=2.24\right)$.

\begin{tabular}{|c|c|c|}
\hline Taxa & Riqueza de especies (S) & Diversidad $\left(\mathrm{H}^{\prime}\right)$ \\
\hline \multirow{2}{*}{ Plantas } & $46(\mathrm{BPQ})$ & $3.34(\mathrm{BPQ})$ \\
\cline { 2 - 3 } & $43(\mathrm{BR})$ & $3.12(\mathrm{BR})$ \\
\hline \multirow{2}{*}{ Aves } & $74(\mathrm{BR})$ & $3.92(\mathrm{BR})$ \\
\hline \multirow{2}{*}{ Moluscos } & $72(\mathrm{BSI})$ & $3.86(\mathrm{BSI})$ \\
\cline { 2 - 3 } & $31(\mathrm{BP})$ & $2.87(\mathrm{BP})$ \\
\cline { 2 - 3 } & $23(\mathrm{BR})$ & $2.24(\mathrm{BFL})$ \\
\hline
\end{tabular}

6. De acuerdo a nuestras observaciones, los bosques riparios son el uso de suelo más conservado y más biodiverso en el área de estudio, posiblemente en todo el país.

7. La mayor riqueza de especies, diversidad y equitatividad en general se encontró asociada a aquellos usos de tipo boscoso como bosques riparios, bosques primarios y bosques secundarios intervenidos.

8. No obstante, las cercas vivas en el caso de las aves, y los tacotales, en el caso de los moluscos, presentan una alta riqueza, diversidad y equitatividad.

9. En cuanto a la estructura de la vegetación, se encontró una correlación muy significativa $(\mathrm{r}=0.344, \mathrm{p}<0.01)$ entre la diversidad y la diversidad estructural, es decir, la complejidad del follaje.

10. Estos resultados indican que la diversidad estructural del follaje debe ser tenida en cuenta conjuntamente con la cobertura, para hacer inferencias sobre la diversidad de otros grupos en los sistemas silvopastoriles.

\section{Agradecimientos}

Este proyecto ha sido financiado por ABC-Gaia y el Banco Mundial. Agradecemos a los Drs. Rob Chipley y George Wallace, de $\mathrm{ABC}$, el apoyo que nos han brindado en todo momento en relación con aspectos metodológicos y/o logísticos, lo que ha permitido el desarrollo exitoso de la primera fase del proyecto. Al Lic. Elías Ramírez, Coordinador nacional del proyecto, por su apoyo en todos los aspectos y a la Lic. Aura Yorlene Cárdenas por los mapas de fincas que han servido de base para el desarrollo de nuestro trabajo. Pedro Argeñal y Herty Betancourt, todos de NITLAPAN UCA, por su apoyo en el trabajo de campo.

Queremos extender nuestro agradecimiento a la tesista del componente de aves, Sandra 
Hernández, a los tesistas del componente de moluscos Xochlit Duarte y Lenin Aburto, así como a los estudiantes Andrés Largaespada, César Olivar y Eddy Rizo, de la carrera de Ingeniería en Calidad Ambiental de la Universidad Centroamericana (UCA) que han colaborado con el procesamiento de la información.

\section{Referencias bibliográficas}

-ALTONAGA, K. (1988). Estudio taxonómico y biogeográfico de las familias Endodontidae, Euconulidae, Zonitidae y Vitrinidae (Gastropoda: Pulmonata: Stylommatophora) de la península ibérica, con especial referencia al País Vasco y zonas adyacentes. Tesis Doctoral (inédita). Universidad del País Vasco.

-AOU (1998). Check-List of North American Birds. USA. Allen Press $7^{\mathrm{ma}}$ ed.

-BERGIN, T.M., BEST, L.B., FREEMARK, K.E. y KOEHLER, K.J. (2000). "Effects of Landscape Structure on Nest Predation in Roadsides of a Midwestern Agroecosystem: a Multiscale Analysis". Landscape Ecology, 15:131-143.USA.

-CBM-MARENA (2001). Información CBM Nicaragua. MARENA-SICA/CCAD-PNUD/GEF-GTZPNUMA-BANCO MUNDIAL. Managua. En soporte CD.

-CCAD (1999). Lista de fauna de importancia para la conservación en Centroamérica y México: listas rojas, listas oficiales y especies en apéndices CITES. WWF, UICN, SICA. -CHIPLEY, R., WALLACE, G. y NARANJO, L. (2003). Manual para el monitoreo de Biodiversidad. American Bird Conservacy, Washington DC. Mimeo.

-COLWELL, R.K. y CODDINGTON, J.A. (1994). "Estimating Terrestrial Biodiversity Through Extrapolation”. Philosophical Transactions of the Royal Society (Series B), 345:101118.USA.

-ESTRADA, A. y COATES-ESTRADA, R. (2002). “Dung Beetles in Continuous Forest, Forest Fragments and in an Agricultural Mosaic Habitat Island at Los Tuxlas, Mexico. Biodiversity and Conservation, 11:1903-1918.USA.

-ESTRADA, A., R. COATES-ESTRADA, R. y MERRIT, D.A. (1997). "Anthropogenic Landscape Changes and Avian Diversity at Los Tuxlas, Mexico". Biodiversity and Conservation, 6:19-43.USA.

-FOURNIER, E. y LOREAU, M. (2001). "Respective Roles of Recent Edges and Forest Patch Remnants in the Maintenance of Ground-Beetle (Coleoptera: Carabidae) Diversity in an Agricultural Landscape". Landscape Ecology, 16:17-32.USA.

-HARRIS, L.D. (1984). The Fragmented Forest. Island Biogeographic Theory and the Preservastion of Biotic Diversity. University of Chicago Press, Chicago, Illinois.

-HILL, M.O. (1973). "Diversity of Evenness: a Unifying Notation and its Consequences". Biology, 54: 321-346.USA.

-HOWELL, S. y WEBB, S. (1995). A Guide to the Birds of México and Northern Central América. Oxford University press Inc. New York, USA.

-INEC (1995). Censos Nacionales. Cifras oficiales finales Managua, Instituto Nacional de Estadísticas y Censos, INEC.

-INIFOM. En línea. http::// www.inifom.gob.ni/ Caracterizaciones.php-19k. Consultada 4.11.2003.

-JACCARD, P. (1901). Etude comparative de la distribution florale dans une portion des Alpes et des Jura. Bull. Soc. Vaudoise Sci. Nat., 37:547-579. 
-JAMES, F.C. y SHUGART, H.H. (1970). «A Quantitative Method of Habitat Description”. Audubon Field Notes, 24:727-736.USA.

-JEANNERET, P., SCHÜPBACH, B., PFIFFNER, L. y WALTER, T. (2003). "Arthropod Reaction to Landscape Changes and Avian Features in Agricultural Landscapes". Landscape Ecology, 18:253-263.USA.

-LEVARD, L., MARÍN, Y. y NAVARRO, I. (2001). "Municipio de Matiguás, potenciales y limitantes del desarrollo agropecuario”. Cuaderno de Investigación, No.11, Managua, UCA Publicaciones, Universidad Centroamericana.

-LUDWIG, J.A. y REYNOLDS, J.F. (1988). Statistical Ecology: a Primer on Methods and Computing. A Wiley Interscience Publication. USA.

-MACARTHUR, R.H. y MACARTHUR, J.W. (1961). On Bird Species Diversity. Ecology, 42(3):594598.USA.

-MARENA (2001). Informe del ambiente en Nicaragua 2003. II informe GEO. Impresión Comercial, Managua, La Prensa.

-MARULL, J. y MARSALLACH, J.M. (2002). La conectividad ecológica en el área metropolitana de Barcelona. Ecosistemas, (URL: http://aeet.org/ecosistemas022/ibvestigacion6.htm).

164 Consultada 05, 2002.

-MORALES, D., CHAVES, M. y ROCHA, L. (2002). Análisis del cambio de cobertura arbórea en una microcuenca del Río Bulbul, Matiguás, Nicaragua, para los años 1954, 1968, 1981 y 1987. Turrialba, Costa Rica. Mimeo.

-MURCIA, C. (1995). "Edge Effect in Fragmented Forest: Implications for Conservation". Tree, 10(2):58-62).USA.

-NARANJO, L.G. En Línea. Wild birds in Latin American pasture lands. http:// www.virtualcentre.org. Consultada 18.05.04, 2:05 pm.

-NOON, B.R. (1981). "Techniques for Sampling Avian Habitats". Pp. 42-52 en The Use of Multivariate Statistics in Studies of Wildlife Habitat (E. E. Capen, ed.). USDA Forest Serv. Tech. Rep., RM9-87.USA.

-PÉREZ, A.M. (2001). Biodiversidad: aspectos conceptuales y datos sobre Nicaragua y América Central. Managua, Gaia, 1:1-40.

-PÉREZ, A.M. (2002). Redes ecológicas: un uso alternativo del término y su aplicación a la conservación de la diversidad in situ. Un enfoque preliminar. Managua, Gaia, 2: 1-10.

-PÉREZ, A.M. (2004). Aspectos conceptuales, análisis numérico, monitoreo y publicación de datos sobre biodiversidad. Managua, Araucaria-Marena.

-PEREZ, A.M. y LOPEZ, A. (2002). Atlas de los moluscos continentales del Pacífico de Nicaragua. Managua, Editorial UCA.

-PÉREZ, A.M. y TORRES, S. (2003). Clave taxonómica malacológica. Managua, Asociación Gaia. En CD (Soporte digital).

-PÉREZ, A.M., VILASECA, J.C. y ZIONE, N. (1996). Sinecología básica de moluscos terrestres en cuatro formaciones vegetales de Cuba. Rev. Biol. Trop., 44(1):133-146. Cuba. -PÉREZ, A.M., BORNEMANN, G., CAMPO, L., ARANA, I., SOTELO, M., RAMÍREZ, F., y CASTAÑEDA, E. (2003). Biodiversidad y producción en sistemas silvopastoriles. Cuadernos de Investigación, Managua, UCA Publicaciones.

-PÉREZ, A.M., SOTELO, M. y ARANA I. (2004). "Altitudinal Variation of Diversity on Landsnail Communities from Maderas Volcano, Ometepe, Nicaragua". Iberus 22(1):133-145.

-PETTT, S. y USHER, M.B. (1998). "Biodiversity in Agricultural Landscapes: the Ground Beetle Communities of Woody Uncultivated Habitats". Biodiversity and Conservation, 7:1549-1561.USA. 
-PIELOU, E.C. (1977). Mathematical Ecology. New Cork, New York. -POVEDA, L. y SANCHEZ-VINDAS, P. (1999). Árboles y palmas del Pacífico Norte de Costa Rica. Claves dendrológicas. Heredia, Costa Rica. Editorial Guayacán. -PUENTE, A.I. (1994). Estudio taxonómico y biogeográfico de la superfamilia helicoidea Rafinesque, 1815 (Gastropoda: Pulmonata: Stylommatophora) de la península Ibérica e Islas Baleares. Tesis Doctoral, Universidad del País Vasco. Mimeo.

-RADULOVICH, R. (1994). Tecnologías productivas para sistemas agrosilvopecuarios de ladera con sequía estacional. Serie Técnica, Informe Técnico No. 222, Turrialba, Costa Rica. Centro Agronómico Tropical de Investigación y Enseñanza (CATIE).

-REMANE, A., STORCH, V. y WELSCH, U. (1980). Zoología sistemática. Barcelona. Ediciones Omega.

-RICKETTS, T.H., DAILY, G.C., EHRLICH, P.R. y PAY, J.P. (2001). "Countryside Biogeography of Moths in a Fragmented Landscape: Biodiversity in Native and Agricultural Habitats". Conservation Biology, 15(2):378-388.USA.

-SALAS, J. (1993). Árboles de Nicaragua. Managua, IRENA.

-SAUNDERS, D.A., HOBBS, R.J. y MARGULES, C.R. (1991). "Biological Consequences of Ecological Fragmentation". Conservation Biology, 5(1):18-32.USA.

-SCHEMSKE, D.W. BROKAW, N. (1981). Treefalls and the Distribution of Understorey Birds in a Tropical Forest. Ecology, 62:938-945.USA.

-SHANNON, C.E. y WEAVER, W. (1949). The Mathematical Theory of Communication. Urbana, Illinois. University Illinois Press.

-SIMPSON, E.H. (1949). Measurement of Diversity. Nature, 163:688.USA.

-SOKAL, R.R. y ROLHF, F.J. (1981). The Principles and Practice of Statistics in Biological Research. New York. State University of New York at Stony Brook.

-SOLEM, A. (1974). The Shell Makers. New York. John Wiley \& Sons.

-SOLEM, A. y CLIMO, F.M.(1985). "Structure and Habitat Correlations of Sympatric New Zealand Land Snail Species". Malacología 26(1-2):1-30.

-STILES, F.G. y SKUTCH, A. (1998). Guía de aves de Costa Rica. Heredia, Costa Rica. INBIO, $2^{\mathrm{a}}$ ed.

-TANSLEY, A.G. y CHIPP T. F. (1926). Aims and Method in the Study of Vegetation. Br. Emp. Veg. Comm., London, Whitefriars Press.

-WCMC. (1992). Global Diversity. Status of the Earth's Living Resources. London, Chapman \& Hall.

-WEIBULL, A.C., OSTMAN, O. y GRANQUIST, A. (2003). "Species Richness in Agroecosystems: the Effect of Landscape, Habitat and Farm Management. Biodiversity and Conservation, 12:1335-1355.USA.

-WILCOX, B.A. (1980). "Insular Ecology and Conservation". En: M.E. SOULÉ y WILCOX, B.A. eds, pp. 95-117. Conservation Biology, an Evolutionary-Ecological Perspective. Massachussets, Sinauer, Sunderland.

-WUNDERLE, J.M. (1994). "Métodos para contar aves terrestres del Caribe" USDA, Forest Service. General Technical Report SO-100. USA.

-WUNDERLE, J.M. y WAIDE, R.B. (1993). "Distribution of Overwintering Nearctic Migrants in the Bahamas and Greater Antilles". Condor, 95:904-933.USA. 